Pacific Journal of Mathematics

A SHAPE THEORY WITH SINGULAR HOMOLOGY 


\title{
A SHAPE THEORY WITH SINGULAR HOMOLOGY
}

\author{
FRIEDRICH W. BAUER \\ Alexander Dinghas in memoriam
}

\begin{abstract}
A modified concept of a shape of a topological space is introduced which allows some basic geometric constructions: (1) One has a convenient homotopy concept which originates from a cylinder functor. (2) All inclusions of compact metric spaces are cofibrations. (3) Shape mappings which agree on the intersection of their counterimages can be pasted together (existence of push-outs). (4) There exists a singular complex $\bar{S}$ which has the same properties for shape mappings as the ordinary singular complex $S$ for continuous maps. (5) Consequently one has a singular (shape) homology which for compact metric spaces turns out to be isomorphic to the (shape-theoretically defined) homotopical homology (in the sense of G. W. Whitehead) and to the Steenrod-Sitnikov homology.
\end{abstract}

0. Introduction. Two topological spaces $X$ and $Y$ are supposed to be of the same shape whenever they have similar geometric properties. In order to give this concept a precise meaning one has to define an appropriate shape category $S$ with topological spaces as objects but with a new class of morphisms (other than continuous mappings or their homotopy classes) the so called shape mappings. This category should permit most of the geometric constructions which constitute the value of the category of $C W$-spaces, to be performed.

The first model of a shape category was introduced 1968 by K. Borsuk. Four years later S. Mardešić [7] gave a rather simple characterization of Borsuk's shape category $S$. Roughly speaking $S$ turned out to be the universal category (with topological spaces as objects) in which two spaces $X, Y$ are equivalent whenever they cannot be distinguished by those homotopy invariants which are determined by mappings of $X$ resp. $Y$ into arbitrary $C W$-spaces. Thus Čech cohomology is (but singular homology is not) a shape invariant.

In the present paper we present a modified shape category $\overline{\boldsymbol{K}}$ which has the following property:

Let $X=X_{1} \cup X_{2}, D=X_{1} \cap X_{2}$ and $f_{t} \in \overline{\boldsymbol{K}}\left(X_{t}, Y\right), i=1,2$ be morphisms which coincide on $D$, then there exists a unique $f \in \bar{K}(X, Y)$ which restricts to $f_{i}$ on $X_{t}$ (Lemma 3.1, 3.2, Proposition 3.3). In other words: Mappings can be pasted together.

Although $\overline{\boldsymbol{K}}$ is not a homotopy category, it does have a natural notion of homotopy so one can define fibrations and cofibrations within 
$\overline{\boldsymbol{K}}$. In particular, under some restrictions imposed on the relevant category of topological spaces, every inclusion turns out to be a cofibration (Theorem 3.8, Theorem 3.10). There exist nontrivial singular simplexes in $\overline{\boldsymbol{K}}$, therefore we can define a singular complex $\bar{S}(X)$ which although generally not equivalent to $S(X)$, nevertheless exhibits all properties of the ordinary singular complex $S(X)$ (Proposition 3.5, Theorems 3.6, 4.7). In particular $\bar{S}(X)$ gives rise to a new kind of singular homology

$$
H_{n}^{\text {sin }}(X ; \boldsymbol{E})=H_{n}(|\bar{S}(X)| ; \boldsymbol{E})
$$

where $\boldsymbol{E}=\left\{E_{n} \mid n \in Z, \nu_{n}: \Sigma E_{n} \rightarrow E_{n+1}\right\}$ is a spectrum [4] and $|\cdot|$ denotes Milnor's geometric realization of a Kan set.

On the other hand one can perform in $\overline{\boldsymbol{K}}$ all the constructions that lead to homology groups in the sense of G. W. Whitehead [11]:

$$
\bar{H}_{n}(X ; \boldsymbol{E})=\lim _{k}\left[S^{n+k}, X \wedge E_{k}\right] .
$$

Theorem 5.4 asserts that these two homology theories are isomorphic.

We have a functor $h:$ Top $\rightarrow \overline{\boldsymbol{K}}$, hence each functor $\phi: \overline{\boldsymbol{K}} \rightarrow \boldsymbol{T}$ $(\boldsymbol{T}=$ any category) gives rise to a functor $\phi h:$ Top $\rightarrow \boldsymbol{T}$.

In particular $\tilde{H}_{*}$ can be considered as a homology functor on Top, and one can therefore attempt to recognize $\bar{H}_{*}$ as one of the already known, classical homology functors. This is settled in $\S 7$ where $\bar{H}_{*}$ is shown to be isomorphic to the Steenrod-Sitnikov homology theory [1], [10] $H_{*}^{S}$ on the category of connected compact metric pairs (Theorem 7.7). This functor $H_{*}^{s}$ itself is one of the most important homology concepts in algebraic topology: In 1951 Sitnikov established his version of the Alexander-Pontrjagin duality theorem [1] for arbitrary subsets of $S^{n}$. However the homology needed for this theorem turned out to be isomorphic (up to a shift of dimension) to a contruction of $N$, Steenrod [10], given in 1940. Recently it was discovered that a homology construction by A. A. Kolmogoroff from 1936 is also isomorphic to $\boldsymbol{H}_{*}^{s}$ [8]. Thus, although $H_{*}^{s}$ does not enter into any textbook on algebraic topology, it is in fact not only one of the most important but also one of . the oldest homology concepts in topology, moreover $H_{*}^{S}$ is the universal exact homology which originates from Cech homology [2].

For the proof of Theorem 7.7. We avoid an explicit construction of $H_{*}^{S}$ and use Milnor's axiomatic characterization [8]; in fact this is the reason that we have to confine ourselves to a category of compact metric spaces.

Theorem 7.7 in combination with Theorem 5.4 expresses the fact 
that the Steenrod-Sitnikov-homology is both the intrinsic singular homology in the shape category $\overline{\boldsymbol{K}}$ and simultaneously the homotopyhomology theory in the sense of G. W. Whitehead. Finally we have evidently a concept of homotopy groups $\pi_{*}(X)$ in $\overline{\boldsymbol{K}}$ and a Hurewicztheorem (Theorem 5.6) with $\bar{H}_{*}$ as the canonical homology in $\overline{\boldsymbol{K}}$.

The construction of $\overline{\boldsymbol{K}}$ is very similar to that of Mardešic for Borsuk's shape category: We simply replace ordinary categories by 3 -categories, taking into account the changes which are consequences of this modification. The abstract procedure (feasable for any pair of 3-categories $\boldsymbol{P} \subset \boldsymbol{K}$ ) is outlined in $\S 2$, while $\S 1$ contains some well known facts from topology and category theory which are indispensable for the following sections. In $\S \S 3,4$ we provide some geometric constructions such as that of smash-product, of push-outs, cofibrations and of the singular complex together with proofs of the related theorems. Singular homology and homotopy groups are introduced in $\$ 5$, and we prove that Whitehead's homology [11] is isomorphic to singular homology; though this assertion already holds in Top it has a different meaning in Top, the corresponding shape category.

$\$ 6$ is devoted to the construction of a second kind of a singular complex functor $\bar{S}^{\prime}$ (which is homotopy equivalent to $\bar{S}$ ). This section is of an entirely auxiliary character.

The isomorphism theorem 7.7 is prepared, stated and proved in $\S \S 7$ and 8 .

Although the reader is not assumed to be familiar with any explicit construction of $H_{*}^{S}$ (as we pointed out already everything is deduced from J. Milnor's axiomatic characterization) the following remark might be useful as background information: For compact metric spaces Čech homology is isomorphic to Vietoris homology [1] which operates with "true cycles". Such a "true cycle" is related to a "Sitnikov cycle" in formally the same way as a Borsuk-Mardešic shape mapping is to a shape mapping in our category $\overline{\boldsymbol{K}}$. On the other hand there exists a formal connection between the two different shaping constructions and the problem of developing a good stable category in stable homotopy theory: The Borsuk-Mardešić category starts from the homotopy category Top just as the Spanier-Whitehead $S$-category, while the more effective stabilizations (see, e.g. [4] for further reference) pass to the homotopy category only after stabilization has been effected.

1. Preliminaries. In this section we collect some facts from category theory and general topology which are relevant for our subject:

(a) 2-categories: The reader is assumed to be familiar with the definition of a 2-category and a 2-functor [4]. A category $K$ is a 2-category whenever it carries the following additional structure: for any $X, Y \in \boldsymbol{K}, \boldsymbol{K}(X, Y)$ is again a category and the induced maps 


$$
\boldsymbol{K}(f, Y)=f^{*}: \boldsymbol{K}(X, Y) \longrightarrow \boldsymbol{K}\left(X^{\prime}, Y\right)
$$

for $f \in \boldsymbol{K}\left(X^{\prime}, X\right)$ resp.

$$
\boldsymbol{K}(X, g)=g_{*}: \boldsymbol{K}(X, Y) \longrightarrow \boldsymbol{K}\left(X, Y^{\prime}\right)
$$

for $g \in K\left(Y, Y^{\prime}\right)$ are functors on $\boldsymbol{K}(X, Y)$. The morphisms $f \in \boldsymbol{K}(X, Y)$ are called 1-morphisms and the morphisms in the category $K(X, Y)$ (for fixed $X, Y)$ are called 2-morphisms. We will henceforth simply write $\omega f$ resp. $g$ instead of $\boldsymbol{K}(f, Y)(\omega)$ resp. $\boldsymbol{K}(X, g)(\omega)$ for any 2-morphism $\omega \in \boldsymbol{K}(X, Y)$. Since the diagram

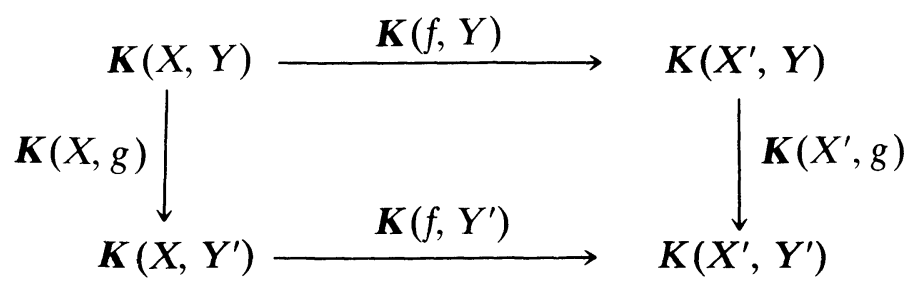

is commutative, we have

$$
g(\omega f)=(g \omega) f
$$

where $\omega \in \boldsymbol{K}(X, Y)(r, s)$ and $r, s \in \boldsymbol{K}(X, Y)$.

A 2-functor $\phi: \boldsymbol{K} \rightarrow \boldsymbol{L}$ for two 2-categories is an ordinary functor such that the assignment

$$
\begin{gathered}
\boldsymbol{K}(X, Y) \longrightarrow \boldsymbol{L}(\phi(X), \phi(Y)) \\
f \longmapsto \phi(f)
\end{gathered}
$$

is a functor; in particular this implies the existence of a transformation of the 2-morphisms. Moreover this functor is supposed to have the following properties:

$$
\begin{aligned}
& \phi(g \omega)=\phi(g) \phi(\omega), \quad \omega \in \boldsymbol{K}(X, Y)(r, s) \\
& \phi(\omega f)=\phi(\omega) \phi(f)
\end{aligned}
$$

for $f, g$ as above.

The most important examples of 2-categories originate from categories of categories (with functors as morphims and functor transformations as 2-morphisms) and categories with homotopy. This second kind of example will be basic for the rest of this paper. 
(b) 2-categories and homotopy: We will assume once for all in this paper, that all 2-morphisms are isomorphims (but of course not necessarily identities). In all those cases where this is not true ab initio we form the appropriate quotient categories $\boldsymbol{K}(X, Y) / \boldsymbol{K}(X, Y)$ (thus we convert all maps into isomorphisms [3]). The homotopy category $\boldsymbol{K}_{h}$ for a 2-category $\boldsymbol{K}$ is an ordinary category which has the same objects as $\boldsymbol{K}$ but classes $[f]$ of maps which are equivalent in the category $K(X, Y)$ $(f \in \boldsymbol{K}(X, Y))$ as morphisms. Any 2 -functor $\phi: \boldsymbol{K} \rightarrow \boldsymbol{L}$ resp. any transformation of 2-functors $\varphi: \phi \rightarrow \Psi$ induces a functor

$$
\phi_{h}: \boldsymbol{K}_{h} \rightarrow \boldsymbol{L}_{h} \quad \text { resp. } \quad \varphi_{h}: \phi_{h} \rightarrow \Psi_{h}
$$

In any 2-category we have the concept of a fibration and of a cofibration.

Definition 1.1. (a) A map $p: E \rightarrow B$ in $\boldsymbol{K}$ is a fibration if for any $f_{0}, f_{1}: X \rightarrow B, \tilde{f}_{0}: X \rightarrow E$, and $\varphi: f_{0} \rightarrow f_{1}$ with

$$
p \tilde{f}_{0}=f_{0}
$$

there exists a $\tilde{f}_{1}: X \rightarrow E$, and $\tilde{\varphi}: \tilde{f}_{0} \rightarrow \tilde{f}_{1}$ such that

$$
p \tilde{\varphi}=\varphi \text {. }
$$

(b) A map $i: B \rightarrow E$ is a cofibration if for any $\tilde{f}: E \rightarrow X$ in $K$, and $\varphi: f i \rightarrow f_{1}$, there exists a $f_{1} \in \boldsymbol{K}(E, X)$, and $\tilde{\phi}: \tilde{f} \rightarrow f_{1}$ such that

$$
\tilde{\varphi} i=\varphi \text {. }
$$

We will return to this notion of a cofibration very soon. The most interesting example of a 2-category is Top:

(c) The category Top as a 2-category: The category Top carries the structure of a 2-category in the following form: Let $f_{0}, f_{1} \in$ Top $(X, Y)$, then a homotopy $H$ from $f_{0}$ to $f_{1}$ is a mapping $H: X \times$ $[0, a] \rightarrow Y$ for suitable $a \geqq 0$ such that $H(x, 0)=f_{0}, H(x, a)=f_{1}$. Let $H^{\prime}: X \times[0, b] \rightarrow Y$ be a homotopy from $f_{1}$ to $f_{2}$ (which implies $H(x, a)=$ $H^{\prime}(x, 0)$ for any $\left.x \in X\right)$; then we define a composition $H^{\prime} \cdot H: X \times$ $[0, a+b] \rightarrow Y^{\circ}$ by

$$
H^{\prime} \circ H(x, t)=\left\{\begin{array}{l}
H(x, t) \cdots t \leqq a \\
H^{\prime}(x, t-a) \cdots t \geqq a .
\end{array}\right.
$$

This yields a homotopy from $f_{0}$ to $f_{2}$. The trivial homotopy 


$$
\begin{aligned}
& 1_{f}: X \times[0,0] \longrightarrow Y \quad f \in \operatorname{Top}(X, Y) \\
& (x, 0) \longmapsto f(x)
\end{aligned}
$$

operates evidently as the identity. We can summarize:

Proposition 1.2. The previous construction makes Top into a 2-category (with the H's as 2-morphisms).

REMARK. We can of course reformulate the whole construction for

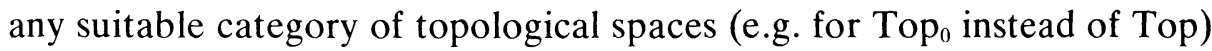
and also for the category $\mathscr{S}_{E}$ of Kan-complexes. There exists an alternative way of defining the 2-morphisms in Top (which is equivalent to the preceding one): If we agree to identify (the formal 2-morphisms) $H^{-1}$ with $H(x, a-t)$ and accept the "cancellation law" $H \cdot H^{-1}=$ $H^{-1} \circ H=1$, then every 2-morphism (which originally is a class of words of homotopies according to the construction of a quotient category [3]), turns out to be represented by a single homotopy.

(d) Mappings having the homotopy extension property (HEP) for polyhedra: an inclusion $i: A \subset B$ in Top has the HEP (with respect to a space $X)$ if for any map $f: B \rightarrow X$ and for any homotopy $H: A \times$ $[0, s] \rightarrow X, H(a, 0)=f i$ there exists a homotopy $\tilde{H}: B \times[0, s] \rightarrow X$ such that $\tilde{H} \mid A \times[0, s]=H, H(b, 0)=f, b \in B$. A map $i$ is a cofibration if $i$ has the HEP for any $X \in$ Top. This is of course in accordance with definition 1.1.b.

Let $i$ : $A \subset B$ be any inclusion of a closed subset $A$ into $B$ and let $(\boldsymbol{K}, \boldsymbol{P})$ be any of the following pairs of categories, where $\boldsymbol{P}$ is a full subcategory of $\boldsymbol{K}$ :

(1) $\boldsymbol{K}=$ metric spaces, $\boldsymbol{P}=C W$-spaces.

(2) $\boldsymbol{K}=$ paracompact spaces, $\boldsymbol{P}=$ locally finite polyhedra.

(3) $\boldsymbol{K}=$ binormal spaces (i.e. $X$ and $X \times I$ are normal), $\boldsymbol{P}=$ finite polyhedra. We have the following assertion:

THEOREM 1.3. Let $(\boldsymbol{K}, \boldsymbol{P})$ be any of the three pairs mentioned above, then any inclusion $i: A \subset B$ for closed $A$ in $K$ has the $H E P$ for any $P \in P$.

Proof. The theorem is classical [5]. In any of these cases we have the following situation: Let $f: A \rightarrow P$ be a mapping in $\boldsymbol{K}, P \in \boldsymbol{P}$, then there exists an open neighborhood $U$ of $A$ in $B$ and an extension $\hat{f}: U \rightarrow P$ of $f$. Now the HEP for $P$ is equivalent to the following extension property: For any map $f: B \times 0 \cup A \times I \rightarrow P$ there exists an extension $F: B \times I \rightarrow P$. This extension can be established in the fol- 
lowing way: Extend $f$ over an open neighborhood $U$ of $B \times 0 \cup A \times I$ in $B \times I$, and find a neighborhood $V \supset A$ with $V \times I \subset U$; by normality there is a continuous $p: B \rightarrow I$ with $p(A)=1, p(B-V)=0$, and $F(x, t)=\hat{f}(x, t p(x))$ is the desired extension.

(e) For later purposes we need a simple category theoretical concept:

Definition 1.4. Let $\boldsymbol{T}$ be any category. (1) A morphism $\alpha \in$ $\boldsymbol{T}(X, Y)$ is called indecomposable whenever $\alpha=\beta \gamma$ implies that either $\beta$ or $\gamma$ is an identity.

(2) A category $\boldsymbol{T}$ is a strong tree category (st-category) whenever each $\alpha \in \boldsymbol{T}$ allows a unique decomposition into indecomposable elements.

(3) A category $\boldsymbol{T}$ is a tree category whenever there exists an st-subcategory $\boldsymbol{T}^{\prime} \subset \boldsymbol{T}$ and a right adjoint $\phi: T \rightarrow T^{\prime}$ to the inclusion.

In case $\boldsymbol{T}$ is a 2-category, we will always assume that $\boldsymbol{T}^{\prime}$ is a subcategory with all 2-morphisms identities.

The concept of a tree category is basic for our future work. We will frequently use the following simple

Proposition 1.5. Let $\boldsymbol{T}$ be a tree category with $\boldsymbol{T}^{\prime} \subset \mathrm{T}$ as st-category, let $\boldsymbol{K}$ be any category, and let $\boldsymbol{A}: \boldsymbol{T} \rightarrow \boldsymbol{K}$ be an assignment (not necessarily a functor) sending objects to objects and each morphism $f \in \boldsymbol{T}(X, Y)$ to a morphism $A(f) \in \boldsymbol{K}(A(X), A(Y))$. Then there exists a unique functor $\bar{A}: \boldsymbol{T} \rightarrow \boldsymbol{K}$ such that $A=\bar{A}$ on the objects of $\boldsymbol{T}^{\prime}$ and on the undecomposable morphisms of $\boldsymbol{T}^{\prime}$.

Proof. Set $\bar{A}\left(\alpha_{1} \cdots \alpha_{n}\right)=A\left(\alpha_{1}\right) \cdots A\left(\alpha_{n}\right)$ for each composition of undecomposables $\alpha_{1} \cdots \alpha_{n}$. This defines a functor $\bar{A}: \boldsymbol{T}^{\prime} \rightarrow \boldsymbol{K}$ which, by using the right adjoint, is extendable over $\boldsymbol{T}$.

Here we present the following example:

1. Let $Q$ be a fixed Hilbert-cube and let $\boldsymbol{P}$ be the category of all compact ANR, embedded in $Q$ with continuous maps as morphisms. Any compact metric $X$ can be embedded in $Q$. For $X \subset Q$ let $\left\{K_{n}\right\}$, $n=1,2, \cdots$ be the following sequence of compact ANR neighborhoods of $X$ in $Q$ : Set $K_{1}=Q$ and assume $K_{n-1} \subset K_{n-2} \subset \cdots \subset K_{1}$ already defined. We take for any $x \in X$ the family $\{K(x, 1 / n)\}$ of all $1 / n$ neighborhoods of $x$. Since $X$ is compact, there exists a finite subcovering $K\left(x_{1}, 1 / n\right), \cdots, K\left(x_{r}, 1 / n\right)$. Let $K_{n}$ be $\bigcup_{i=1}^{r} \overline{K\left(x_{i}, 1 / n\right)} \cap K_{n-1}$. This is 
certainly an ANR and we have $\bigcap_{n=1}^{\infty} K_{n}=X$. We denote by $i_{n}^{m}: K_{m} \rightarrow K_{n}$, $m \geqq n$ respectively $i_{n}=i_{n}^{X}: X \rightarrow K_{n}$ the inclusion. Observe that $i_{n}^{m}=$ $i_{m}^{m+1} i_{m+1}^{m+2} \cdots i_{n-1}^{n}$.

Now let $f: X \rightarrow P \in \boldsymbol{P}$ be any continuous map, then Dugundji's extension theorem ([5] p. 188, Theorem 6.1) provides us with a canonical extension $f^{\prime}: U \rightarrow P, X \subset U$. Let $n$ be the smallest possible $n$ (consequently $K_{n}$ is the largest possible $K_{n}$ ) for which one can extend $f$ to a $\phi(f): K_{n} \rightarrow P$. If $f=g r, r: P_{1} \rightarrow P_{2}$, then we have $\phi(f): K_{m} \rightarrow P_{2}$, $\phi(g): K_{n} \rightarrow P_{2}$ and $m \geqq n$ and we define $\phi(r)=i_{n}^{m}$. If $f=i_{n}: X \subset K_{n}$, then $\phi(f)=1: K_{n} \rightarrow K_{n}$. We take the category $\boldsymbol{T}^{\prime}$ whose objects are inclusions $i_{n}$ with mappings $i_{n}^{m}$ as morphisms, $m \geqq n$, and the category $\boldsymbol{T}$ with mappings $f: X \rightarrow P \in \boldsymbol{P}$ as objects and commutative triangles $r: f_{1} \rightarrow f_{2}, r f_{1}=f_{2}$ as morphisms. Then the assignment $\phi: \boldsymbol{T} \rightarrow \boldsymbol{T}^{\prime}$ is a functor which is right adjoint to the inclusion $\boldsymbol{T}^{\prime} \subset \boldsymbol{T}$ and $\boldsymbol{T}^{\prime}$ is an st-category.

Details of the proof are simple and left to the reader. The preceding construction works for the category Com of compact metric spaces.

(f) $n$-categories: A category $\boldsymbol{K}$ is an $n$-category whenever $\boldsymbol{K}(X, Y)$ for any $X, Y \in \boldsymbol{K}$ is an $(n-1)$-category and the induced mappings $f^{*}, g_{*}$ (from $\left.(a)\right)$ are $(n-1)$-functors, which render some diagrams commutative. We are not going into the details. Since we

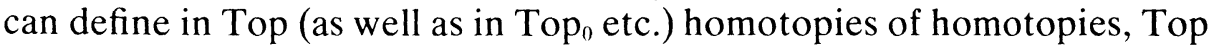
becomes a 3-category. In fact this can easily be iterated giving Top the structure of an $n$-category for arbitrary $n$.

At one place in $\S 2$ there will be need for the following explicit construction for an $n$-category $\boldsymbol{K}$ :

Definition 1.6. The category $\boldsymbol{K}^{(t)}, \quad i=2, \cdots, n$ has $(i-1)$ morphisms as objects and triples $\left(w_{1}, w_{2}, u\right): v_{1} \rightarrow v_{2}$ as morphisms, where $w_{1}, w_{2}, v_{1}, v_{2}$ are $(i-1)$-morphisms such that the diagram

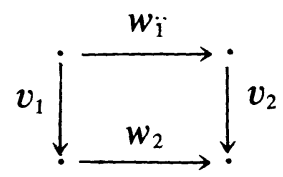

exists (the dots are $(i-2)$-morphisms resp. objects of the category if $i=2)$ and $u$ is an $i$-morphism such that 


$$
u: w_{2} v_{1} \approx v_{2} w_{1}
$$

Composition of morphisms is defined in the following way:

$$
\left(w_{1}^{\prime}, w_{2}^{\prime}, u^{\prime}\right) \circ\left(w_{1}, w_{2}, u\right)=\left(w_{1} w_{1}^{\prime}, w_{2} w_{2}^{\prime}, w_{1}^{\prime} u \circ w_{2} u^{\prime}\right)
$$

This defines a category $\boldsymbol{K}^{(i)}$.

For the sake of completeness we set $\boldsymbol{K}=\boldsymbol{K}^{(1)}$. We assume from now on that all $i$-morphisms for $i>1$ will be isomorphisms. In Definition 2.3 we will explicitely need $\boldsymbol{K}^{(3)}$.

2. The construction of $\overline{\boldsymbol{K}}$. Let $\boldsymbol{P} \subset \boldsymbol{K}$ be a full embedding of one 3-category into another. The category $\overline{\boldsymbol{K}}$ which we are going to construct will have the structure of a 2-category, however the procedure can easily be iterated giving a $n$-category $\overline{\boldsymbol{K}}$ for an arbitrary $n$. As we have already stated, all 2- and 3-morphims will be isomorphisms.

Definition 2.1. For each $X \in \boldsymbol{K}$ we construct a 2-category $\boldsymbol{P}_{X}$ as follows:

(1) The objects: all morphisms in $\boldsymbol{K}, g: X \rightarrow P \in \boldsymbol{P}$.

(2) A morphism $g_{1} \rightarrow g_{2}$ is a pair $(r, \omega)$ where $r \in \boldsymbol{P}\left(P_{1}, P_{2}\right)$ and $\omega: r g_{1} \approx g_{2}$ is a 2-morphism in $\boldsymbol{K}$.

(3) Composition of morphisms:

$$
g_{1} \stackrel{\left(r_{1}, \omega_{1}\right)}{\longrightarrow} g_{2} \stackrel{\left(r_{2}, \omega_{2}\right)}{\longrightarrow} g_{3}
$$

is the morphism

$$
\left(r_{2} r_{1}, \omega_{2} \circ r_{2} \omega_{1}\right): g_{1} \rightarrow g_{3}
$$

(4) A 2-morphism $(\tau, \xi):(r, \omega) \approx\left(r^{\prime}, \omega^{\prime}\right)$ is a pair, where $\tau: r \approx r^{\prime}$ is a 2-morphism in $\boldsymbol{K}$ and $\xi$ a 3-morphism such that

$$
\xi: \omega^{\prime} \circ\left(\tau g_{1}\right) \approx \omega
$$

It is straightforward to verify that this definition does in fact determine a 2-category. Clearly each $\tau$ is an isomorphism because by our standard assumption all 2-morphisms in $\boldsymbol{K}$ are isomorphisms. Evidently the definition of $\boldsymbol{P}_{X}$ is in close relation to Definition 1.6.

Proposition 2.2. Any $f \in \boldsymbol{K}(X, Y)$ gives rise to a 2-functor $\boldsymbol{P}_{f}: \boldsymbol{P}_{Y} \rightarrow \boldsymbol{P}_{X}$ by setting $\boldsymbol{P}_{f}(g)=g f, \boldsymbol{P}_{f}(r, \omega)=(r, \omega f)$, and similarly on the 2-morphisms. These functors $\Lambda=\boldsymbol{P}_{f}$ have the following properties: 
(1) $g \in \boldsymbol{K}(Y, P), P \in \boldsymbol{P} \Rightarrow \Lambda(g) \in \boldsymbol{K}(X, P)$

(2) $(r, \omega) \in \boldsymbol{P}_{Y}\left(g_{1}, g_{2}\right) \Rightarrow \Lambda(r, \omega)=\left(r, \omega_{1}\right)$ for suitable $\omega_{1}$.

The proof is immediate by checking the list of properties required by the definition of a 2-category and of a 2-functor. The 2-morphism $\omega_{1}$ in (2) is clearly $\omega_{1}=\omega f$.

We come now to the category with which we will be mainly concerned in this paper:

Definition 2.3. Let $\boldsymbol{K}$ be the following category:

(1) Objects: Those of $\boldsymbol{K}$.

(2) Morphisms $\bar{f}: X \rightarrow Y:$ 2-functors $\bar{f}=\Lambda: \boldsymbol{P}_{Y} \rightarrow \boldsymbol{P}_{X}$ satisfying conditions (1), (2) of Proposition 2.2.

(3) Composition of morphisms: Composition of functors.

(4) A 2-morphism $\bar{\nu}: \bar{f}_{1} \rightarrow \bar{f}_{2}$ in $\overline{\boldsymbol{K}}$ is an assignment $\bar{\nu}: \boldsymbol{P}_{Y} \rightarrow \boldsymbol{K}^{(3)}$ such that

$$
\bar{\nu}(g): \bar{f}_{1}(g) \approx \bar{f}_{2}(g)
$$

and such that for any $(r, \omega): g_{1} \rightarrow g_{2}, \bar{f}_{i}(r, \omega)=\left(r, \omega_{\imath}\right)$ one has

$$
\bar{\nu}(r, \omega)=\left(\omega_{1}, \omega_{2}, \mu\right): r \bar{\nu}\left(g_{1}\right) \approx \bar{\nu}\left(g_{2}\right)
$$

for a suitable 3-morphism $\mu$ (cf. Definition 1.6) in a functorial way.

REMARK. (1) If $\boldsymbol{K}$ is a 2-category (hence a 3-category in which all 3 -morphisms are identities) a 2 -morphism in $\overline{\boldsymbol{K}}$ is simply a family

$$
\bar{\nu}(g): \bar{f}_{1}(g) \approx \bar{f}_{2}(g)
$$

of 2-morphisms satisfying the naturality condition

$$
\bar{\nu}\left(g_{2}\right) \circ \omega_{1}=\omega_{2} \circ r \bar{\nu}\left(g_{1}\right) .
$$

(2) The condition (1) is strictly speaking a cosequence of the second, because $\bar{f}(r, \omega)=\left(r, \omega^{\prime}\right)$ makes sense only if (1) is fulfilled. It may happen that $r=1: P_{1} \rightarrow P_{2}$ and therefore we can compose $\left(1, \omega_{2}\right) \circ\left(1, \omega_{1}\right)=\left(1, \omega_{2} \circ \omega_{1}\right)$, for two 2-morphisms $\omega_{1}, \omega_{2}$. This implies in particular that $\Lambda$ behaves functorially on the 2-morphisms in $\boldsymbol{K}$.

Proposition 2.4. Definition 2.3 determines a 2-category. There exists a 2-functor $h: \boldsymbol{K} \rightarrow \overline{\boldsymbol{K}}$ which is the identity on the object and 


$$
h(f)(g)=g f, \quad g \in \boldsymbol{K}(X, P)
$$

on the morphisms.

The proof is immediate.

To aid the reader in keeping the morphisms of this category in mind the following diagram scheme (not necessarily commutative) may be useful:

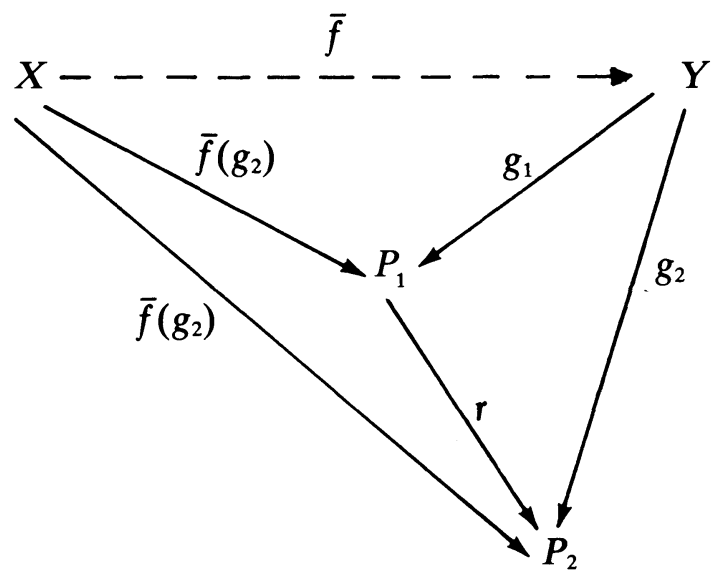

It is essential to observe that $r g_{1}$ may equal $g_{2}$ (so that the morphism $(r, \omega): g_{1} \rightarrow g_{2}$ has as second component the identity 2-morphism in $\boldsymbol{K}$ but this does not automatically imply that

$$
r \bar{f}\left(g_{1}\right)=\bar{f}\left(g_{2}\right),
$$

becauce one must consider the well defined morphism $\omega^{\prime}: r \bar{f}\left(g_{1}\right) \approx \bar{f}\left(g_{2}\right)$ which may not be trivial.

The reader who is familiar with K. Sitnikov's definition of his homology [1] will easily see the analogy.

We have the subclasses:

$$
\begin{aligned}
& \overline{\boldsymbol{K}}_{\boldsymbol{P}}=\{\bar{f} \in \overline{\boldsymbol{K}}(X, P) \mid P \in \boldsymbol{P}, X \in \boldsymbol{K}\} \subset \overline{\boldsymbol{K}} \\
& \boldsymbol{K}_{\boldsymbol{P}}=\{f \in \boldsymbol{K}(X, P) \mid P \in \boldsymbol{P}, X \in \boldsymbol{K}\} \subset \boldsymbol{K}
\end{aligned}
$$

and an assignment

$$
\begin{aligned}
h^{\prime}: \overline{\boldsymbol{K}}_{\boldsymbol{P}} & \longrightarrow \boldsymbol{K}_{\boldsymbol{P}} \\
\bar{f} \longmapsto & \longrightarrow \bar{f}(1), \quad 1 \in \boldsymbol{K}(P, P) .
\end{aligned}
$$


Proposition 2.5. For fixed $X \in \boldsymbol{K}$, and $\boldsymbol{P} \in \boldsymbol{P}, h^{\prime}$ is a functor $h^{\prime} \mid \overline{\boldsymbol{K}}(X, P): \overline{\boldsymbol{K}}(X, P) \rightarrow \boldsymbol{K}(X, P)$. Moreover $h^{\prime} \mid \overline{\boldsymbol{P}}_{X}: \overline{\boldsymbol{P}}_{X} \rightarrow \boldsymbol{H}_{X}, X \in \overline{\boldsymbol{K}}$ is a 2-functor (where $\overline{\boldsymbol{P}}_{X}$ is constructed as $\boldsymbol{P}_{X}$ after $\boldsymbol{P}, \boldsymbol{K}$ have been replaced by $\overline{\boldsymbol{P}}$, $\overline{\boldsymbol{K}})$. For any $f \in \boldsymbol{K}(X, P)$ one has

$$
h^{\prime} h(f)=f
$$

and for any $\bar{f} \in \overline{\boldsymbol{K}}(X, P)$

$$
h h^{\prime}(\bar{f}) \approx \bar{f} .
$$

If $f_{1}, f_{2} \in \boldsymbol{K}(X, P), h\left(f_{1}\right) \approx h\left(f_{2}\right)$, then we have $f_{1} \approx f_{2}$.

Proof. The functorial properties of $h^{\prime}$ are immediate. Let $f \in$ $\boldsymbol{K}(X, P)$, then we have

$$
f \stackrel{h}{\longrightarrow}(g \longmapsto g f) \stackrel{h^{\prime}}{\longrightarrow} h(f)(1)=f
$$

Let $e=h h^{\prime}(\bar{f}), \bar{f} \in \boldsymbol{K}(X, P)$, then evaluation of $e$ on a fixed $g \in \boldsymbol{P}_{\boldsymbol{P}}$ leads to the natural 2-morphism

$$
e(g)=g \bar{f}(1) \approx \bar{f}(g),
$$

which proves (2).

Finally let $f_{1}, f_{2} \in \boldsymbol{K}(X, P), h\left(f_{1}\right) \approx h\left(f_{2}\right), g \in \boldsymbol{P}_{\boldsymbol{P}}$, then we have

$$
g f_{1}=h\left(f_{1}\right)(g) \approx h\left(f_{2}\right)(g)=g f_{2} .
$$

Putting $g=1$ into this equivalence, we get a proof of $f_{1} \approx f_{2}$. As an immediate consequence of 2.5 we have:

THEOREM 2.6. The homotopy categories $\boldsymbol{P}_{h}$ and $\overline{\boldsymbol{P}}_{h}$ are equivalent.

REMARK. (1) Let $\boldsymbol{P}=$ the homotopy category of $C W$-spaces considered as the full subcategory of the homotopy category $\boldsymbol{K}=$ Top $_{h}$, both as trivial 2-categories with no 2-morphisms other than identities; then $\overline{\boldsymbol{K}}$ is easily seen to be Borsuk's shape category [7].

(2) Even in the case $\boldsymbol{P}=\boldsymbol{K}$ the preceding construction gives something new.

The general construction of $\overline{\boldsymbol{K}}$ can be achieved in two steps: First we 
form the category $\boldsymbol{K}_{1}=\overline{\boldsymbol{K}}$ for $\boldsymbol{K}=\overline{\boldsymbol{P}}$. Then we apply the "shaping construction" in the sense of [7] applied to general categories (rather than only to the category of topological spaces). In fact the general construction of [7] is the special case of our construction in which all 2-morphisms are identities (and $\boldsymbol{P}$ is arbitrary).

(3) In order to avoid clumsy notation we will in most cases avoid explicit mention of the functor $h$. For example if $f \in \overline{\boldsymbol{K}}(X, Y)$ and $r \in \boldsymbol{K}(Y, Z)$ then we will simply write $r f \in \overline{\boldsymbol{K}}(\boldsymbol{X}, Z)$ instead of $h(r) f$. This will never cause any ambiguity.

\section{Push-outs, the singular complex and cofibrations.} In this section we deal with the category of topological spaces $\boldsymbol{K}=$ Top or Top $_{o}$ (based spaces) and the corresponding subcategory of $C W$-spaces without (with) basepoints. Our first aim is to show that mappings in $\overline{\boldsymbol{K}}$ which fit together on the intersection of their counterimages can be pasted together:

\section{LEMMA 3.1. If}

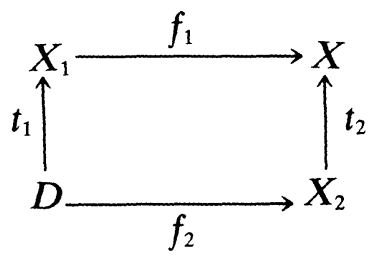

is a push-out diagram in $\boldsymbol{K}$, then the image of (1) under $h: \boldsymbol{K} \rightarrow \overline{\boldsymbol{K}}$ is a push-out diagram in $\overline{\boldsymbol{K}}$.

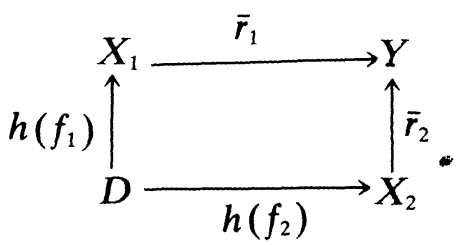

For a fixed $g \in \boldsymbol{K}(Y, P), P \in \boldsymbol{P}$ we get a unique $s: X \rightarrow P$ in $\boldsymbol{K}$ such that

$$
\bar{r}_{1}(g) h\left(f_{1}\right)=h\left(s t_{1} f_{1}\right)=h\left(s t_{2} f_{2}\right)=\bar{r}_{2}(g) h\left(f_{2}\right) .
$$

Hence $\bar{d}(g)=s$ seems to be a candidate for a $\bar{s} \in \overline{\boldsymbol{K}}(X, Y)$ : Let $g \in \boldsymbol{K}(Y, P), g^{\prime} \in \boldsymbol{K}(Y, Q), d \in \boldsymbol{K}(P, Q)$ and $\omega: d g \simeq g^{\prime}$, then we can lift $\omega$ to a unique $\omega^{\prime}: d s(g) \simeq s\left(g^{\prime}\right)$ becauce of the following observation: 
We have

$$
X=X_{1} \cup X_{2} / \sim
$$

where $x_{1} \sim x_{2}$ whenever $f_{1}(d)=x_{1}$ for some $d \in D$. The connecting map $\bar{s}(g): X \rightarrow P$ has the following explicit form:

$$
\bar{s}(f)\left(x_{1}\right)=\bar{r}_{1}(g)\left(x_{1}\right), \quad \bar{s}\left(g^{\prime}\right)\left(x_{2}\right)=\bar{r}_{2}(g)\left(x_{2}\right),
$$

analogously

$$
\bar{s}\left(g^{\prime}\right)\left(x_{1}\right)=\bar{r}_{1}\left(g^{\prime}\right)\left(x_{1}\right), \quad \bar{s}\left(g^{\prime}\right)\left(x_{2}\right)=\bar{r}_{2}\left(g^{\prime}\right)\left(x_{2}\right) .
$$

Hence we have

$$
\begin{aligned}
& d \bar{s}(g) t_{1}=d \bar{r}_{1}(g) \simeq \bar{r}_{1}\left(g^{\prime}\right) \\
& d \bar{s}(g) t_{2}=d \bar{r}_{2}(g) \simeq \bar{r}_{2}\left(g^{\prime}\right),
\end{aligned}
$$

where the homotopy comes from $\bar{r}_{1}(d, \omega)=\left(d, \omega_{1}\right)$ resp. $\bar{r}_{2}(d, \omega)=\left(d, \omega_{2}\right)$.

Because of the commutativity of (1) and (2) these homotopies respect the equivalence relation in $X_{1} \cup X_{2}$ and we obtain a homotopy

$$
\omega^{\prime}: d \bar{s}(g) \simeq \bar{s}\left(g^{\prime}\right)
$$

This $\omega^{\prime}$ is certainly natural and makes $\bar{s}: X \rightarrow Y$ a morphism in $\overline{\boldsymbol{K}}$ by defining

$$
\bar{s}(d, \omega)=\left(d, \omega^{\prime}\right)
$$

The 2-morphisms $\tau:(r, \omega) \rightarrow\left(r^{\prime}, \omega^{\prime}\right)$ are settled in the same way. The verification of the functorial properties of $\bar{s}$ as well as the proof of (1), (2) in 2.2 is routine.

We have $\bar{s} h\left(t_{t}\right) h\left(f_{t}\right)=\bar{r}_{t} h\left(f_{t}\right)$; if $\bar{s} \in \overline{\boldsymbol{K}}(X, Y)$ is any other morphism with this property, the uniqueness of the connecting map (which is part of the definition of a push-out diagram) shows $\tilde{s}=\tilde{s}^{\prime}$. This proves the Lemma.

Lemma 3.1 is basic for numerous constructions in $\overline{\boldsymbol{K}}$. Moreover we can immediately extend it to an arbitrary number of morphisms:

Lemma 3.2. Let $\bar{f}_{\alpha}: D \rightarrow X_{\alpha}$ be any family of morphisms in $\overline{\boldsymbol{K}}$ 
$\alpha \in J \quad$ (= any indexing set) $t_{\alpha}: X_{\alpha} \rightarrow X$ a push-out of the $f_{\alpha}$, then this property remains true after application of $h$.

This yields at once

"Pasting" Proposition 3.3. Let $X=\cup X_{\alpha} \in$ Top, $\bar{f}_{\alpha} \in \boldsymbol{K}\left(\bar{X}_{\alpha}, Y\right)$ any family of morphisms such that $\bar{f}_{\alpha}\left|X_{\alpha} \cap X_{\tau}=\bar{f}_{\tau}\right| X_{\alpha} \cap X_{\tau}$, then there exists a unique $\bar{f} \in \bar{K}(X, Y)$ with $\bar{f} \mid X_{\alpha}=\bar{f}_{\alpha}$.

Here, by any abuse of notion, we write $\bar{f} \mid A$ for $\bar{f} h(i), i: A \subset X$.

Though the construction of $\bar{S}(X)$ is completely analogous to that of the singular complex $S(X)$ for $X \in$ Top, these are not in general isomorphic.

A singular simplex $\bar{\sigma}^{n} \in \bar{S}(X)_{n}$ is a map $\bar{\sigma}^{n} \in \overline{\boldsymbol{K}}\left(\Delta^{n}, X\right)$ where $\Delta^{n}$ is the standard simplex.

Definition 3.4. For each $X \in \bar{K}$ the singular complex $\bar{S}(X)$ consists of all singular simplexes with face and degeneracy operators defined in the usual way. If $\bar{f} \in \overline{\boldsymbol{K}}(X, Y), \quad \bar{\sigma}^{n} \in \bar{S}(X)$, we set $\bar{f}\left(\bar{\sigma}^{n}\right)=$ $\bar{f} \bar{\sigma}^{n} \in \bar{S}(Y)$.

Recalling that every category with homotopy can be regarded as a 2-category (cf. 1.c), we can summarize some basic facts about this construction:

Proposition 3.5. (a) $\bar{S}$ : Top $\rightarrow \mathscr{S}_{E}$ is a covariant 2-functor. (b) There is a natural transformation $\nu_{X}^{\prime}: S(X) \rightarrow \bar{S}(X)$ by setting $h\left(\bar{\sigma}^{n}\right)(g)=$ $\bar{\sigma}^{n} g, \bar{\sigma}^{n} \in S(X) X \in \bar{K}$. (c) Let $|\cdot|: \mathscr{S}_{E} \rightarrow$ Top be J. Milnor's geometric realization and $\nu=\left|\nu^{\prime}\right|:|S| \rightarrow \bar{S}$, then there exist natural transformations

$$
\omega:|S| \longrightarrow 1_{\text {Top }}, \quad \bar{\omega}:|\bar{S}| \longrightarrow 1_{\text {Top }}
$$

such that

$$
\tilde{\omega} \circ \nu=h(\omega)
$$

Proof. The functorial properties of $\bar{S}$ follow exactly as the corresponding assertions for $S$ [3], [6]. The definition of $\nu^{\prime}: S \rightarrow \bar{S}$ given in the proposition clearly yields a natural transformation of functors. The transformations $\omega:|S| \rightarrow 1, \bar{\omega}:|\bar{S}| \rightarrow 1$ (where of course the first already belongs to the classical theory) are both constructed in the same way: For each nondegenerate simplex $\bar{\sigma}^{n}: \Delta^{n} \rightarrow X$ in $\bar{S}(X)$ we have a cell $\left|\bar{\sigma}^{n}\right| \subset$ $|\bar{S}(X)|$ in the cell structure of $|\bar{S}(X)|$ induced by the realization 
functor. This cell $\left|\bar{\sigma}^{n}\right|$ is mapped by $\bar{\sigma}^{n}$ into $X$. Now we can apply 3.3 and paste together all these morphisms, obtaining a morphism $\bar{\omega}:|S(X)| \rightarrow X$. The contention (4) is now an immediate consequence of the construction of $\left|\nu^{\prime}\right|=\nu, \omega$ and $\bar{\omega}$.

THEOREM 3.6. On $\overline{\boldsymbol{P}} \subset \overline{\boldsymbol{K}}$ the transformation $\overline{\boldsymbol{\omega}}_{p}:|S(P)| \rightarrow$ P yields a homotopy equivalence. Furthermore for any $\bar{f} \in \overline{\boldsymbol{K}}(P, X)$, and $P \in \boldsymbol{P}$ we have up to homotopy a factorization:

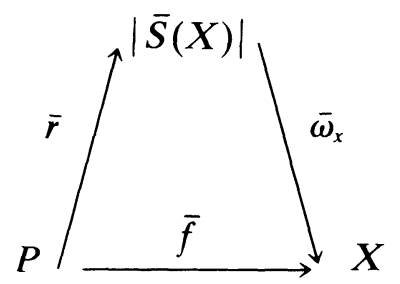

In other words: Every morphism starting from a $P \in \boldsymbol{P}$ in $\overline{\boldsymbol{K}}$ factors in the homotopy category over $|\bar{S}(X)|$.

For the proof of Theorem 3.6 we need a lemma:

LEMMA 3.7. For each $X \in \boldsymbol{P}$. the natural tranformation $\nu:|S(X)| \rightarrow|\bar{S}(X)|$ is a homotopy equivalence. In fact, assuming this Lemma, 3.6 can be established as follows: For any $P \in \boldsymbol{P}$ we have the commutative diagram:

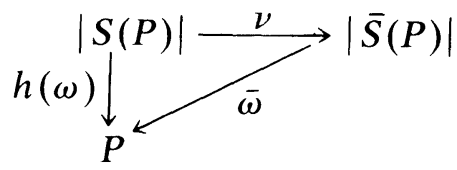

where $\nu$ (by Lemma 3.7) and $\omega$ (by a well known classical argument) are homotopy equivalences.

Since $h$ is a 2-functor, the morphism $h(\omega)$ is a homotopy equivalence and therefore, so also is $\bar{\omega}$.

The existence of a diagram (5) is established by standard arguments: We have the commutative diagram

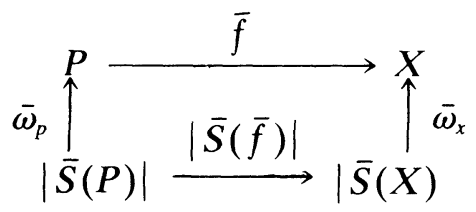

Any_homotopy inverse $\bar{g}: P \rightarrow|\bar{S}(P)|$ of $\bar{\omega}_{p}$ provides us with an $\bar{r}: P \rightarrow|\bar{S}(X)|$ in $\bar{K}$ and then 


$$
\bar{r}=|\bar{S}(\bar{f})| \bar{g}
$$

fits into (5). It remains therefore to establish the Lemma.

Proof of Lemma 3.7. Let $\bar{\sigma}^{n} \in \bar{S}(H)$, then we have a $\mu^{\prime}\left(\bar{\sigma}^{n}\right)=$ $\bar{\sigma}^{n}(1) \in S(P)$. This assignment determines a simplicial mapping and furthermore a natural transformation $\mu^{\prime}: \bar{S} \rightarrow S($ on $\overline{\boldsymbol{P}}$ ). Thus

$$
\left|\mu^{\prime}\right|=\mu:|\bar{S}| \longrightarrow|S|
$$

is a natural transformation on $\overline{\boldsymbol{P}}$. Since $\mu^{\prime}$ on $\bar{\sigma}^{n}: \Delta^{n} \rightarrow X$ is simply $h^{\prime}$, we deduce from $h^{\prime} \circ h=1$ that $\mu \nu=1$.

On the other hand let $\bar{\sigma}^{n} \in \overline{\boldsymbol{K}}\left(\Delta^{n}, P\right)$ be any singular simplex in $\bar{S}(P)$. Using the transformation $\nu^{\prime}$ of Proposition 3.5, we find for any $g \in \boldsymbol{H}(P, Q)$ that we have

$$
\nu^{\prime} \mu^{\prime}\left(\bar{\sigma}^{n}\right)(g)=g \tilde{\sigma}^{n}(1) \in \boldsymbol{K}\left(\Delta^{n}, Q\right) .
$$

Because $h h^{\prime} \approx 1$ (Proposition 2.5) we obtain a natural homotopy between $\left|\bar{\sigma}^{n}\right|$ and $\left|\nu^{\prime} \mu^{\prime}\left(\bar{\sigma}^{n}\right)\right|$, which commutes with the cell structure in $|\tilde{S}(P)|$. We can paste together these homotopies, accomplishing a homotopy between $\nu \mu$ and $1:|\bar{S}(P)| \rightarrow|\bar{S}(P)|$. This completes the proof of Lemma, 3.7, thereby establishing Theorem 3.6.

One of the basic properties of $C W$-complexes is the result that every cellular inclusion is a cofibration. This is no longer true for arbitrary topological spaces. In order to prove a cofibration theorem for $\overline{\boldsymbol{K}}$ we have to impose the following two restrictions on the categories $\boldsymbol{P}$ and $\boldsymbol{K}$ :

$\boldsymbol{P} 1) \quad \boldsymbol{P}$ resp. $\boldsymbol{K}$ are subcategories of $\boldsymbol{P}_{1}$ resp. $\boldsymbol{K}_{1}$, where $\left(\boldsymbol{K}_{1}, \boldsymbol{P}_{1}\right)$ is one of the three pairs listed at the beginning of $\S 1$ (d).

$\boldsymbol{P} 2$ ) For each $X \in \boldsymbol{K}$ (see $\S 2$ ) $\boldsymbol{P}_{X}$ is a tree category (Definition 1.3).

Then we establish

THEOREM 3.8. Under the conditions $\boldsymbol{P} 1)$ and $\boldsymbol{P}$ 2) any closed inclusion $i: A \subset B$ in $\boldsymbol{K}$ is a cofibration in $\overline{\boldsymbol{K}}$ (i.e. $h(i)$ is a cofibration in the sense of Definition 1.1. (b)).

Proof. (1) Equivalent to Theorem 3.8 is the following contention: The inclusion

$$
i: A \times I \cup B \times 0 \subset B \times I
$$


is a coretraction in $\overline{\boldsymbol{K}}$. In other words there exists a morphism $\bar{F}: B \times$ $I \rightarrow A \times I \cup B \times 0$ in $\overline{\boldsymbol{K}}$ which is the identity on $A \times I \cup B \times 0$. Here $I$ is the unit interval.

We shall now establish (1).

(2) Let $g \in \boldsymbol{K}(A \times I \cup B \times 0, P), \quad P \in \boldsymbol{P}$ be any map, then Theorem 1.3 gives an extension $F_{g}: B \times I \rightarrow P$ of $g$, write $X=$ $A \times I \cup B \times 0 ; \quad$ let $g_{\imath} \in \boldsymbol{K}\left(A \times I \cup B \times 0, P_{\imath}\right) \quad i=1,2$ and let $(r, \omega): g_{1} \rightarrow g_{2}$ be a morphism in $\boldsymbol{P}_{X}$. Consider the inclusion

$$
i^{\prime}: X^{\prime}=(A \times I \cup B \times 0 \cup B \times 1) \times I \cup B \times I \times 0 \subset B \times I \times I ;
$$

for the same reason as before, we obtain a mapping

$$
H_{(r, \omega)}=H_{1}: B \times I \times I \longrightarrow P_{2}
$$

such that

$$
H_{1} \mid A \times I \times I \cup B \times I \times 0=\omega
$$

(where we assume without loss of generality that $\omega$ is itself a homotopy (see $\$ 1(c)))$. Since

$$
\begin{aligned}
& H_{1} \mid B \times 0 \times I=r F_{g_{1}} \\
& H_{1} \mid B \times 1 \times I=F_{g_{2}},
\end{aligned}
$$

this construction provides us with a morphism:

$$
\left(r, H_{1}\right): r F_{g_{1}} \longrightarrow F_{g_{2}} \text {. }
$$

However this does not necessarily give a functor $\bar{F}: \boldsymbol{H}_{X} \rightarrow \boldsymbol{H}_{B \times I}$ (i.e. a morphism $\bar{F}: B \times I \rightarrow X$ in $\overline{\boldsymbol{K}}$ ) which extends the identity on $X=$ $A \times I \cup B \times 0$, because we cannot expect functoriality.

(3) To rectify this we need condition $\boldsymbol{P} 2$ ): There exists a strong tree category $\boldsymbol{P}_{X}^{\prime} \subset \boldsymbol{P}_{X}$ and a functor $\phi: \boldsymbol{P}_{X} \rightarrow \boldsymbol{P}_{X}^{\prime}$ which is right adjoint to the inclusion. We then carry out the constructions in (2) for $\boldsymbol{P}_{X}^{\prime}$ : To each indecomposable $(r, \omega)$ we get a $H_{(r, \omega)}$ and (since each morphism in $\boldsymbol{P}_{X}^{\prime}$ allows a unique decomposition into indecomposable factors) we can make the construction functorial for $\boldsymbol{P}_{X}^{\prime}$. On the other hand we have a transformation $\varphi_{g}: \phi(g) \rightarrow g$ (we omit the inclusion $\boldsymbol{P}_{X}^{\prime} \subset \boldsymbol{P}_{X}$ from our notation) and consequently we can define 


$$
\bar{F}(g)=\phi_{g} \bar{F}^{\prime}(\phi(g))
$$

where $\bar{F}^{\prime}$ is the functor on $\boldsymbol{P}_{X}^{\prime}$.

For any $(r, \omega): g_{1} \rightarrow g_{2}$ we define

$$
\bar{F}(r, \omega)=\left(r, \omega_{1}\right) \quad r \bar{F}\left(g_{1}\right) \rightarrow \bar{F}\left(g_{2}\right)
$$

in the following way:

Let $\phi(r, \omega)=(\tilde{r}, \tilde{\omega}), \tilde{\omega}: \tilde{r} \phi\left(g_{1}\right) \simeq \phi\left(g_{2}\right)$ and $\bar{F}^{\prime}(\tilde{r}, \tilde{\omega})=\left(\tilde{r}, \tilde{\omega}_{1}\right)$

$$
\tilde{\omega}_{1}: \tilde{r} \tilde{F}^{\prime}\left(\phi\left(g_{1}\right)\right) \simeq \bar{F}^{\prime}\left(\phi\left(g_{2}\right)\right) .
$$

We then have

$$
\rho_{g_{2}}\left(\tilde{\omega}_{1}\right): \rho_{g_{2}} \tilde{r} \bar{F}^{\prime} \phi\left(g_{1}\right) \simeq \rho_{g_{2}} \bar{F}^{\prime}\left(\phi\left(g_{2}\right)\right) \text {. }
$$

However because of the adjointness of $\phi$ and the inclusion $\boldsymbol{P}_{X}^{\prime} \subset \boldsymbol{P}_{X}$, $\varphi: \phi \rightarrow 1$ is a natural transformation. Therefore there exists a natural homotopy

$$
\varphi_{g_{1}} \bar{r} \approx r \varphi_{g_{2}}
$$

which finally provides us with a homotopy

$$
\omega_{1}: r \bar{F}\left(g_{1}\right) \approx \bar{F}\left(g_{2}\right) \text {. }
$$

For the 2-morphisms in $\boldsymbol{P}_{X}$ we proceed analogously. Since this construction is clearly functorial Theorem 3.8 is proved.

Observe that the third part of the proof is essentially an application of 1.5 in the topological case. In fact this third part can be reformulated more generally as follows:

Lemma 3.9. Let $\boldsymbol{P}_{X}$ be any category satisfying $\left.\boldsymbol{P} 2\right)$ and let $\boldsymbol{P}_{X}^{\prime} \subset \boldsymbol{P}_{X}$ be the corresponding st-category. Then for any 2-functor $f^{\prime}: P_{X}^{\prime} \rightarrow P_{Y}, X$, $Y \in \boldsymbol{K}$ there exists an extension over $\boldsymbol{P}_{X}$ yielding $\bar{f} \in \overline{\boldsymbol{K}}(Y, X)$.

We can use Theorem 3.8 to establish a cofiber theorem for several different pairs of categories. The verifications are in all cases similar. We will confine ourselves to the following case:

Theorem 3.10. Let $\boldsymbol{K}=\mathbf{C o m}$ be the category of compact metric spaces and $\boldsymbol{P}$ the subcategory of all polyhedra. Then every inclusion $i: A \subset B$ in $\boldsymbol{K}$ is a cofibration in $\overline{\boldsymbol{K}}$. 
The theorem follows immediately from the following

Lemma 3.11. For any $X \in \boldsymbol{K}=\mathbf{C o m}$ the category $\boldsymbol{P}_{X}$ is a tree category.

Proof. This is established in precisely the same way in which we proved that the category $\boldsymbol{T}$ in $\S 1$ (e) is a tree category with the notations used there. The subcategory $T^{\prime} \subset \boldsymbol{P}_{X}$ is the same as that for T. Because the pair $(\boldsymbol{K}, \boldsymbol{P})$ is contained in all three pairs listed in $\S 1(\mathrm{~d})$, we deduce that $\boldsymbol{P} 1$ ) is fulfilled. Lemma 3.11 is thereby proved.

This also completes the proof of Theorem 3.10.

Finally the following question will be of some importance in the beginning of the next section:

Let $\boldsymbol{P}^{\prime} \subset$ Top be the full subcategory of all spaces having the homotopy type of a $C W$-complex. We recall that the category $\overrightarrow{\boldsymbol{K}}$ was originally constructed rel. to $\boldsymbol{P}$. We will prove that $\overline{\boldsymbol{K}}$ can also be regarded as being achieved from $\boldsymbol{P}^{\prime} \supset \boldsymbol{P}$. To this end let $\bar{f} \in \overline{\boldsymbol{K}}(X, Y), \bar{f}: \boldsymbol{P}_{Y} \rightarrow \boldsymbol{P}_{X}$ a 2-functor. We are now attempting to extend $\bar{f}$ to a functor

$$
\bar{f}^{\prime}: \boldsymbol{P}_{Y}^{\prime} \longrightarrow \boldsymbol{P}_{X}^{\prime}
$$

in the following way: For a given $P \in \boldsymbol{P}^{\prime}$ we have the homotopy equivalence $\omega_{P}:|S(P)| \rightarrow P$ hence a homotopy inverse $\eta_{P}$ of $\omega_{P}$. The latter is in general not canonical, however there can be fixed a homotopy $L_{P}: \omega_{P} \circ \eta_{P} \simeq 1$.

We define

$$
\bar{f}^{\prime}(g)=\omega_{P} f\left(\eta_{P} g\right)
$$

for $g \in \boldsymbol{P}(Y, P), P \in \boldsymbol{P}^{\prime}$.

Letting $g_{\imath} \in \boldsymbol{K}\left(Y, P_{\imath}\right), P_{\imath} \in \boldsymbol{P}^{\prime}$ and $(r, \omega): g_{1} \rightarrow g_{2}$ we then have a homotopy

$$
\left(\eta_{2} r \omega_{1}\right)\left(\eta_{1} g_{1}\right) \simeq\left(\eta_{2} g_{2}\right)
$$

where we simply write $\eta_{1}$ instead of $\eta_{P_{1}}$ etc. This shows

$$
\left(\eta_{2} r \omega_{1}\right) \bar{f}\left(\eta_{1} g_{1}\right) \simeq \bar{f}\left(\eta_{2} g_{2}\right) .
$$

Finally (by multiplying with $\omega_{2}$ ) we obtain a homotopy

$$
r \circ \omega_{1} \bar{f}\left(\eta_{1} g_{1}\right)=r \circ \bar{f}^{\prime}\left(g_{1}\right) \simeq \omega_{2} \bar{f}\left(\eta_{2} g_{2}\right)=\bar{f}^{\prime}\left(g_{2}\right) .
$$


On the basis of these constructions the proof of the following assertion is technical and straightforward:

Proposition 3.12. Every $\bar{f} \in \overline{\boldsymbol{K}}(X, Y)$ (where $\overline{\boldsymbol{K}}$ is constructed rel. $\boldsymbol{P}=$ category of $C W$-spaces) can be extended to a morphism of $\overline{\boldsymbol{K}}$ (constructed rel. $\boldsymbol{P}^{\prime}$ ). In other words: If we replace $\boldsymbol{P}$ by $\boldsymbol{P}^{\prime}$ we obtain isomorphic categories $\bar{K}$.

This assertion is proved by simply checking that the $\bar{f}^{\prime}$ constructed above is indeed a functor. Details are left to the reader. The value of 3.12 lies in the fact that in the future we can quite freely turn from $\boldsymbol{P}$ to $\boldsymbol{P}^{\prime}$ and vice versa.

It should be observed that the isomorphism mentioned in 3.12 is not necessarily unique (because of the choices of $\eta_{P}$ and of $L_{P}$ involved in the construction).

4. The smash-product in $\overline{\boldsymbol{K}}$. In this section all spaces and mappings are with basepoints. Our aim is the definition of a smashproduct

$$
\bar{f}_{1} \wedge \bar{f}_{2}: X_{1} \wedge X_{2} \rightarrow Y_{1} \wedge Y_{2} \text {, for } \bar{f}_{i}: X_{i} \rightarrow Y_{i}, i=1,2
$$

where $X_{1}, Y_{2}$ are in $\boldsymbol{P}$.

This can be accomplished by establishing a smash-product $\bar{f} \wedge 1: X \wedge Z \rightarrow Y \wedge Z \quad$ for $\bar{f} \in \overline{\boldsymbol{K}}(X, Y), \quad Z \in \boldsymbol{P} \quad$ (resp. $1 \wedge \bar{f} \in \overline{\boldsymbol{K}}$ $(Z \wedge X, Z \wedge Y))$ : for if we assume that $X_{1}, X_{2} \in P$ then we can obtain $\bar{f}_{1} \wedge \bar{f}_{2} \in \bar{K}\left(X_{1} \wedge X_{2}, Y_{1} \wedge Y_{2}\right)$ by the composition

$$
X_{1} \wedge X_{2} \underset{1 \wedge \bar{f}_{2}}{\longrightarrow} X_{1} \wedge Y_{2} \underset{\bar{f}_{1} \wedge 1}{\longrightarrow} Y_{1} \wedge Y_{2}
$$

Now the construction of $\bar{f} \wedge 1_{Z}$ can be reduced to the case $Z=n$-cell, since every $Z \in \boldsymbol{P}$ can be built up by successively attaching $n$-cells, $n=0,1, \cdots$ and we can then apply the "pasting lemma" (Proposition 3.3) to construct $\bar{f} \wedge 1_{Z}$ for arbitrary $Z \in \boldsymbol{P}$.

Thus we need only define $\bar{f} \wedge 1_{Z}$, whenever $Z=n$-cell.

Lemma 4.1. Every morphism $g \in \boldsymbol{K}(Y \wedge Z, P), P \in \boldsymbol{P}$ can be factored in natural way (with respect to $g$ ) over a suitable $g^{\prime} \wedge 1: Y \wedge Z \rightarrow Q \wedge Z$.

Proof. Let $g^{\prime}: Y \rightarrow P^{Z}$ be the transposed of $g, e: P^{Z} \wedge Z \rightarrow P$ the evaluation map 


$$
(\varphi, z) \in P^{Z} \wedge Z \longmapsto \varphi(z)
$$

then one has

$$
e\left(g^{\prime} \wedge 1\right)=g
$$

Due to a well known result by $\mathrm{J}$. Milnor, $P^{z}$ has the homotopy type of a $C W$-space (cf. 3.12). The naturality is immediate.

We are now prepared for:

Definition 4.2. Let $\bar{f} \in \overline{\boldsymbol{K}}(X, Y)$ and let $Z$ be an $n$-cell. Then

$$
\left.\left(\bar{f} \wedge 1_{z}\right)(g)=e\left(\bar{f} g^{\prime}\right) \wedge 1_{z}\right)
$$

The naturality of the factorization in Lemma 4.1 ensures that $\bar{f} \wedge 1_{Z} \in$ $\overline{\boldsymbol{K}}(X \wedge Z, Y \wedge Z)$.

By the preceding discussion we have established a smash-product

$$
\bar{f}_{1} \wedge \bar{f}_{2} \in \boldsymbol{K}\left(X_{1} \wedge X_{2}, Y_{1} \wedge Y_{2}\right)
$$

for $\bar{f}_{\imath} \in \overline{\boldsymbol{K}}\left(X_{\imath}, Y_{1}\right), i=1,2, X_{1}, Y_{2} \in \boldsymbol{P}$.

The main properties of this product given in the following lemmas, are analogous to those of the smash-product in $\boldsymbol{P}$.

Lemma 4.3. Let $f \in \boldsymbol{K}(X, Y)$ and $Z \in \boldsymbol{P}$. Then

$$
h(f) \wedge 1_{z}=h\left(f \wedge 1_{z}\right) .
$$

In other words: The functor $h$ is compatible with the smash-product.

Proof. Let $g \in \boldsymbol{K}(Y \wedge Z, P), P \in \boldsymbol{P}$. If $g^{\prime}: Y \rightarrow P^{Z}$ is the transposed map to $g$, then one has

$$
\left(h(f) \wedge 1_{z}\right)(g)=e\left(h(f)\left(g^{\prime}\right) \wedge 1_{z}\right)=e\left(g^{\prime} f \wedge 1\right) .
$$

Now for $(x, z) \in X \wedge Z$ the last map is

$$
\begin{aligned}
e\left(g^{\prime} f \wedge 1\right)(x, z) & =e\left(g^{\prime} f(x), z\right)=g^{\prime}(f(x))(z) \\
& =g(f(x), z)=g(f \wedge 1,(x, z)) ;
\end{aligned}
$$

on the other hand,

$$
h(f \wedge 1))(g)=g(f \wedge 1)
$$

and this completes the proof. 
LeMma 4.4. (a) Let $\bar{f}_{0}, \bar{f}_{1} \in \overline{\boldsymbol{K}}(X, Y) ; \bar{f}_{0} \simeq \bar{f}_{1}, Z \in \boldsymbol{P}$, then

$$
\bar{f}_{0} \wedge 1_{Z} \simeq \bar{f}_{1} \wedge 1_{Z}
$$

(b) Let $\bar{f} \in \overline{\boldsymbol{K}}(X, Y), \quad g=g_{1} \wedge 1 \in \boldsymbol{K}(Y \wedge Z, P \wedge Z), \quad Z \in \boldsymbol{P}$ (i.e. $\left.g_{1} \in \boldsymbol{K}(Y, P)\right)$, then there is a homotopy

$$
(\bar{f} \wedge 1)(g) \simeq f\left(g_{1}\right) \wedge 1, \quad \text { natural } \quad \text { rel. } \quad g
$$

(c) Let $\quad \bar{f}_{\imath} \in \overline{\boldsymbol{K}}\left(X_{\imath}, Y_{\imath}\right), \quad i=1,2, \quad X_{1}, Y_{2} \in \boldsymbol{P}, \quad g=g_{1} \wedge g_{2} \in$ $K\left(Y_{1} \wedge Y_{2}, P_{1} \wedge P_{2}\right)$, then we have a homotopy

$$
\left(\bar{f}_{1} \wedge f_{2}\right)(g) \simeq \bar{f}_{1}\left(g_{1}\right) \wedge \bar{f}_{2}\left(g_{2}\right)
$$

natural with respect to $g_{1}$ and $g_{2}$.

Proof. Ad (a): Using the preceding notations we have

$$
\bar{f}_{0}\left(g^{\prime}\right) \simeq \bar{f}_{1}\left(g^{\prime}\right)
$$

for the transposed $g^{\prime}$ of a $g \in \boldsymbol{K}(Y \wedge Z, P), P \in \boldsymbol{P}$. Thus

$$
\begin{aligned}
\left(\bar{f}_{0} \wedge 1\right)(g) & =e\left(\bar{f}_{0}\left(g^{\prime}\right) \wedge 1\right) \simeq e\left(\bar{f}_{1}\left(g^{\prime}\right) \wedge 1\right) \\
& =\left(\bar{f}_{1} \wedge 1\right)(g) .
\end{aligned}
$$

This induces a homotopy $\bar{f}_{0} \wedge 1 \simeq \bar{f}_{1} \wedge 1$ in $\overline{\boldsymbol{K}}$.

Ad (b): Letting $\xi: P \rightarrow(P \wedge Z)^{Z}$ be the map $\xi(p)=(z \mapsto(p, z))$, $p \in P, z \in Z$, it is easily checked that

$$
\left(g_{1} \wedge 1\right)^{\prime}=\xi g_{1}
$$

Consequently

$$
\begin{aligned}
\left(\bar{f}_{1} \wedge 1\right)(g) & =e\left(\bar{f}\left(\left(g_{1} \wedge 1\right)^{\prime} \wedge 1\right) \simeq e\left(\bar{f}\left(\xi g_{1}\right) \wedge 1\right)\right. \\
& \simeq e\left(\xi \bar{f}\left(g_{1}\right) \wedge 1\right)=\bar{f}\left(g_{1}\right) \wedge 1,
\end{aligned}
$$

the last equality being true because it is seen to be true for each $(x, z) \in X \wedge Z$.

This proof works for $Z=n$-cell and can be extended to any $Z \in \boldsymbol{P}$ using 3.3 as usual.

Ad (c): We have the following sequence of homotopies: 


$$
\begin{aligned}
\left(\bar{f}_{1} \wedge \bar{f}_{2}\right)\left(g_{1} \wedge g_{2}\right) & =\left(\bar{f}_{1} \wedge \bar{f}_{2}\right)\left(1 \wedge g_{2}\right)\left(g_{1} \wedge 1\right) \\
& =\left(\bar{f}_{1} \wedge 1\right)\left(1 \wedge \bar{f}_{2}\right)\left(1 \wedge g_{2}\right)\left(g_{1} \wedge 1\right) \\
& \simeq\left(1 \wedge g_{2}\right)\left(1 \wedge \bar{f}_{2}\right)\left(\bar{f}_{1} \wedge 1\right)\left(g_{1} \wedge 1\right) \\
& \simeq\left(1 \wedge g_{2}\right)\left(1 \wedge \bar{f}_{2}\right)\left(\bar{f}_{1}\left(g_{1}\right) \wedge 1\right)\left(1 \wedge \bar{f}_{2}\left(g_{2}\right)\right)\left(\bar{f}_{1}\left(g_{1}\right) \wedge 1\right)
\end{aligned}
$$

Here we have abbreviated with " 1 " the corresponding identities.

LEMma 4.5. For $\bar{f} \in \overline{\boldsymbol{K}}\left(X, Y_{1} \wedge Y_{2}\right) Y_{2}, X \in \boldsymbol{P}$ we have a homotopy

$$
\omega(\bar{f}):\left(p_{1} \bar{f} \wedge p_{2} \bar{f}\right) d \simeq \bar{f} .
$$

For $\bar{f}=\bar{f}_{1} f, f \in \boldsymbol{H}\left(X, X^{\prime}\right)$ we have

$$
\left(p_{1} \bar{f}_{1} f \wedge p_{2} \bar{f}_{1} f\right) d=\left(p_{1} \bar{f}_{1} \wedge p_{2} \bar{f}_{1}\right) d^{\prime} f
$$

where $d: X \rightarrow X \wedge X, d^{\prime}: X^{\prime} \rightarrow X^{\prime} \wedge X^{\prime}$ are the diagonal maps and $p_{i} \in$ $K\left(Y_{1} \wedge Y_{2}, Y_{1}\right), i=1,2$ are the projections.

Furthermore the following naturality condition holds:

$$
\omega\left(f_{1} f\right)=\omega\left(f_{1}\right) f
$$

Proof. At first we prove (2): One has $d^{\prime} f=(f \wedge f) d$ and $\left(p_{1} \bar{f}_{1} \wedge p_{2} \bar{f}_{1}\right) d^{\prime} f=\left(p_{1} \bar{f}_{1} \wedge p_{2} \bar{f}_{1}\right)(f \wedge f) d$. Therefore to establish (2) it is enough to verify that

$$
\left(p_{1} \bar{f}_{1} f \wedge p_{2} \bar{f}_{1} f\right)=\left(p_{1} \bar{f}_{1} \wedge p_{2} \bar{f}_{1}\right)(f \wedge f) .
$$

We evaluate the left hand side of (3) at a $g \in \boldsymbol{K}\left(Y_{1} \wedge Y_{2}, P\right), P \in \boldsymbol{P}$ :

$$
\begin{aligned}
\left(p_{1} \bar{f}_{1} f \wedge p_{2} \bar{f}_{1} f\right)(g) & =\left(p_{1} \bar{f}_{1} f \wedge 1\right)\left(1 \wedge p_{2} \bar{f}_{1} f\right)(g) \\
& =\left(1 \wedge p_{2} f_{1} f\right)\left(e\left(\left(p_{1} f_{1} f\right)\left(g^{\prime}\right) \wedge 1\right)\right) \\
& =\left(1 \wedge p_{2} f_{1} f\right)\left(e\left(\left(p_{1} f_{1}\right)\left(g^{\prime}\right) f \wedge 1\right) .\right.
\end{aligned}
$$

As for the right hand side we obtain:

$$
\begin{aligned}
\left(p_{1} \bar{f}_{1} \wedge p_{2} \bar{f}_{1}\right)(f \wedge f)(g) & =\left(p_{1} \bar{f}_{1} \wedge 1\right)\left(1 \wedge p_{2} \bar{f}_{1} f\right)(f \wedge 1)(g) \\
& =\left(1 \wedge p_{2} \bar{f}_{1} f\right)\left(e\left(p_{1} \bar{f}_{1}\left(g^{\prime}\right) f \wedge 2\right)\right.
\end{aligned}
$$

This proof works as usual for $Y_{2}=n$-cell and can be extended for an arbitrary $Y_{2}$ by applying 3.3. Relation (2) is thereby proved. 
Proof of (1). Let $g=g_{1} \wedge g_{2} \in \boldsymbol{K}\left(Y_{1} \wedge Y_{2}, P_{1} \wedge P_{2}\right), q_{i} \in \boldsymbol{K}\left(P_{1} \wedge P_{2}, P_{\imath}\right)$ the projections, then from Lemma 4.4 we find

$$
\left(p_{1} \bar{f} \wedge p_{2} \bar{f}\right) d(g)=\left(p_{1} \bar{f} \wedge p_{2} \bar{f}\right)(g) d \simeq\left(\dot{q}_{1} \bar{f}(g) \wedge q_{2} \bar{f}(g)\right) d
$$

however the last mapping is equal to $\bar{f}(g)$ because (1) is true for any map $\bar{f}(g) \in \boldsymbol{K}\left(X, P_{1} \wedge P_{2}\right)$. The general case $\left(g \in \boldsymbol{K}\left(Y_{1} \wedge Y_{2}, P\right)\right)$ follows from Definition 4.2 and 3.3 in the usual way.

Finally the naturality condition for $\omega(f)$ is immediate.

This completes the proof of Lemma 4.5.

As a corollary we have

Lemma 4.6. Let $\bar{f}_{i} \in \overline{\boldsymbol{K}}\left(X, Y_{i}\right), i=1,2, X, Y_{2} \in \boldsymbol{P}, p_{\imath}$ as in 4.5 then we have

$$
\bar{f}_{1} \wedge \bar{f}_{2} \simeq p_{1}\left(\bar{f}_{1} \wedge \bar{f}_{2}\right) d \wedge p_{2}\left(\bar{f}_{1} \wedge \bar{f}_{2}\right) d
$$

where as in 4.5 the homotopy is natural with respect to $\bar{f}_{1}, \bar{f}_{2}$ in an obvious sense.

Proof. We introduce the diagonal map $d^{\prime}: X \wedge X \rightarrow X \wedge X \wedge X$ for the space $X \wedge X$ and deduce from Lemma 4.5 that

$$
\left(p_{1}\left(\bar{f}_{1} \wedge \bar{f}_{2}\right) \wedge p_{2}\left(\bar{f}_{1} \wedge \bar{f}_{2}\right)\right) d^{\prime} \simeq \bar{f}_{1} \wedge \bar{f}_{2} .
$$

On the other hand we have $d^{\prime}=d \wedge d$ and therefore

$$
\begin{aligned}
p_{1}\left(\bar{f}_{1} \wedge \bar{f}_{2}\right) d \wedge p_{2}\left(\bar{f}_{1}\left(\wedge \bar{f}_{2}\right) d\right. & =\left(p_{1}\left(\bar{f}_{1} \wedge \bar{f}_{2}\right) \wedge p_{2}\left(\bar{f}_{1} \wedge \bar{f}_{2}\right)\right)(d \wedge d) \\
& =\left(p_{1}\left(\bar{f}_{1} \wedge \bar{f}_{2}\right) \wedge p_{2}\left(\bar{f}_{1} \wedge \bar{f}_{2}\right)\right) d^{\prime} .
\end{aligned}
$$

Since the naturality can easily be formulated as in Lemma 4.5 the proof is complete.

The following theorem is basic for our work on homology in $\overline{\boldsymbol{K}}$ :

Theorem 4.7. For $X \in \boldsymbol{K}, Y \in \boldsymbol{P}$ there exists a natural homotopy equivalence in $\boldsymbol{P}$ (therefore a fortiori in $\overline{\boldsymbol{P}}$ )

$$
|\bar{S}(X)| \wedge|\bar{S}(Y)| \simeq|\bar{S}(X \wedge Y)| .
$$


Proof. We use the fact that for any $K, L \in \mathscr{S}_{E^{\prime}}|K \wedge L|$ and $|K| \wedge|L|$ are homotopy equivalent, where $K \wedge L=K \times L / K \vee L$ (cf. $[6])$.

What remains is is an assertion (in $\mathscr{S}_{E}$ ) that

$$
\bar{S}(X \wedge Y) \simeq \bar{S}(X) \wedge \bar{S}(Y)
$$

We use the same method as that leading to the corresponding contention for $S$ (instead of $\bar{S}$ ).

(a) There exists a natural transformation

$$
\begin{aligned}
\varphi: \bar{S}(X) \wedge \bar{S}(Y) & \longrightarrow \bar{S}(X \wedge Y) \\
\left(\bar{\sigma}^{n}, \bar{\tau}^{n}\right) & \longrightarrow\left(\bar{\sigma}^{n} \wedge \bar{\tau}^{n}\right) d,
\end{aligned}
$$

$d: \Delta^{n} \rightarrow \Delta^{n} \wedge \Delta^{n}$ the diagonal map. In fact from Lemma 4.5 (2) it follows immediately that the map is compatible with face and degeneracy operators. The smash-product involved in (6) exists by definition. Furthermore $\left(\bar{\sigma}^{n} \wedge \bar{\tau}^{n}\right) d$ is a degeneracy of the basepoint whenever $\bar{\sigma}^{n}, \bar{\tau}^{n}$ are of this form. Hence the natural transformation $\phi$ exists.

(b) We have a natural transformation going into the opposite direction:

$$
\begin{aligned}
\psi: \bar{S}(X \wedge Y) & \longrightarrow \bar{S}(X) \wedge \bar{S}(Y) \\
\bar{\sigma}^{n} & \longrightarrow\left(p_{X} \bar{\sigma}^{n}, p_{Y} \bar{\sigma}^{n}\right)
\end{aligned}
$$

where $p_{X}, p_{Y}$ are the projections.

(c) We have

$$
\psi \varphi\left(\bar{\sigma}^{n}, \bar{\tau}^{n}\right)=\left(p_{X}\left(\bar{\sigma}^{n} \wedge \bar{\tau}^{n}\right) d, p_{Y}\left(\bar{\sigma}^{n} \wedge \bar{\tau}^{n}\right) d\right)
$$

so Lemma 4.6 assures us that there is a natural (i.e. with face and degeneracy operators compatible) homotopy between the right pair in $\bar{S}(X) \wedge \bar{S}(Y)$ and $\left(\tilde{\sigma}^{n}, \bar{\tau}^{n}\right)$. By standard arguments this yields a homotopy between $\psi \varphi$ and the identity. In order to prove the analogous result for $\varphi \psi$ we observe:

$$
\psi \phi\left(\bar{\sigma}^{n}\right)=\left(p_{X} \bar{\sigma}^{n} \wedge p_{Y} \bar{\sigma}^{n}\right) d
$$

From Lemma 4.5 and the preceding argument we deduce

$$
\psi \varphi \simeq 1
$$

This completes the proof of Theorem 4.7. 
Corollary 4.8. Let $\psi:|\bar{S}(X \wedge Y)| \rightarrow|\bar{S}(X)| \wedge|\bar{S}(Y)|$ be the homotopy equivalence from Theorem 4.7 then the following compatibility condition holds:

$$
\bar{\omega}_{X \wedge Y}=\left(\bar{\omega}_{X} \wedge \bar{\omega}_{Y}\right) \circ \psi
$$

The proof is an easy consequence of the construction of $\varphi$ and of $\bar{\omega}_{X \wedge Y}:|S(X \wedge Y)| \rightarrow X \wedge Y$ resp. $\bar{\omega}_{X} \wedge \bar{\omega}_{Y}:|\bar{S}(X)| \wedge|\bar{S}(Y)| \rightarrow X \wedge Y$. Details are left to the reader.

While the construction of the smash-product in $\overline{\boldsymbol{K}}$ is of some delicacy, there occurs no such trouble for the wedge of two maps:

Let $\bar{f}_{i} \in \overline{\boldsymbol{K}}\left(X_{i}, Y_{1}\right), g: Y_{1} \vee Y_{2} \rightarrow P \in \boldsymbol{P}$, we define

$$
\left(\bar{f}_{1} \vee \bar{f}_{2}\right)(g)=\bar{f}_{1}\left(g i_{1}\right) \vee \bar{f}_{2}\left(g i_{2}\right),
$$

where $i_{1}: Y_{1} \rightarrow Y_{1} \vee Y_{2}$ are the inclusions. This produces a mapping $\bar{f}_{1} \vee \bar{f}_{2} \in \overline{\boldsymbol{K}}\left(X_{1} \vee X_{2}, Y_{1} \vee Y_{2}\right)$ which has all properties expected of a wedge. No restrictions on $X_{\imath}, Y_{\imath}$ are necessary. In particular the wedge has the following properties:

Lemma 4.9 . (a) $h\left(\bar{f}_{1}\right) \vee h\left(\bar{f}_{2}\right)=h\left(\bar{f}_{1} \vee \bar{f}_{2}\right), \bar{f}_{1}, \bar{f}_{2} \in \overline{\boldsymbol{K}}$.

(b) Let $\bar{f} \in \bar{K}\left(X_{1} \vee X_{2}, Y\right), j_{k}: X_{k} \rightarrow X_{1} \vee X_{2}, k=1,2$ the inclusions and $\varphi: Y \vee Y \rightarrow Y$ the folding map [3], then we have an equality:

$$
\varphi\left(\bar{f} j_{1} \vee \bar{f} j_{2}\right)=\bar{f}
$$

Proof. Because (a) is trivial, we only prove (b): Let $g \in \boldsymbol{K}(Y, P)$, $P \in \boldsymbol{P}$ be any map, then

$$
\begin{aligned}
\left(\bar{f} j_{1} \vee \bar{f} j_{2}\right)(g) & =\left(\bar{f} j_{1} \vee \bar{f} j_{2}\right)(g \phi) \\
& =\bar{f} j_{1}\left(g \varphi i_{1}\right) \vee f j_{2}\left(g \varphi i_{2}\right) \\
& =f j_{1}(g) \vee f j_{2}(g) \\
& =\bar{f}(g) j_{1} \vee \bar{f}(g) j_{2}=\bar{f}(g) .
\end{aligned}
$$

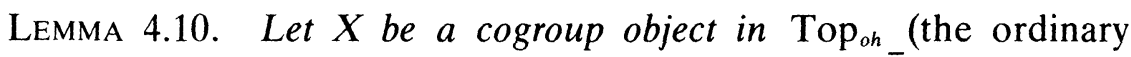
homotopy category), then $X$ is also a cogroup object in $\overline{\boldsymbol{K}}_{h}$.

In particular $\overline{(\mathrm{Top})}_{h}(X, Y)$ carries a natural group structure for any $Y \in$ Top $_{o}$.

The proof is an exact repetition of the corresponding statement for Top $_{h}$ (cf. [3]). 
In the future we will constantly make use of the following assertion:

Proposition 4.11. Two mappings $\bar{f}_{0}, \bar{f}_{1} \in \overline{\boldsymbol{K}}(X, Y)$ are homotopic (in $\overline{\boldsymbol{K}}$ ) if and only if there exists a map in $\overline{\boldsymbol{K}} \bar{F}: X \times I \rightarrow Y$ such that

$$
\bar{F} i_{0}=\bar{f}_{0}, \quad \bar{F} i_{1}=\bar{f}_{1}
$$

where as usual $i, X \subset X \times I$ denotes the standard inclusion $(j=0,1)$.

The proof follows immediately from the Definition 2.3 (4) and the observation that in Top (as well as in Top ${ }_{o}$ ) a 3-morphism is a homotopy of homotopies.

Details are left to the reader.

5. Homotopy groups and singular homology. We deal with the category $\boldsymbol{K}=$ Top $_{o}$ and $\boldsymbol{P}=$ full subcategory of based $C W$ spaces. For any two objects $X, Y \in \boldsymbol{K}$ we define

$$
\begin{aligned}
{[X, Y] } & =\boldsymbol{K}_{h}(X, Y) \\
\bar{\pi}_{n}\left(X, x_{0}\right) & =\left[S^{n}, X\right] .
\end{aligned}
$$

Lemma 4.10 assures that $\bar{\pi}_{n}\left(X, x_{0}\right)=\bar{\pi}_{n}(X)$ is a group for $n>0$ and that $\bar{\pi}_{*}$ is a functor from $\overline{\boldsymbol{K}}$ to the category of graded groups $(n>0)$.

Some facts concerning $\bar{\pi}_{*}$ are summarized in the following

THEOREM 5.1. (a) $\bar{\pi}_{n}(X)$ is abelian for $n>1$.

(b) There exists an isomorphism of functors

$$
\bar{\pi} h \approx \pi \quad \text { on } \quad \boldsymbol{P} .
$$

(c) The transformation $\bar{\omega}:|\bar{S}(\cdot)| \rightarrow 1_{\bar{K}}$ induces an isomorphism

$$
\bar{\pi}_{n}(\bar{\omega}): \bar{\pi}_{n}(|\bar{S}(X)|) \approx \bar{\pi}_{n}(X) .
$$

Proof. Ad (a): This assertion follows from well known properties of $S^{n}, n>1$ which are not affected by the transition from $\boldsymbol{K}$ to $\overline{\boldsymbol{K}}$.

Ad (b): We deduce from 2.6 that $\boldsymbol{P}_{h}$ and $\overline{\boldsymbol{P}}_{h}$ are isomorphic through the isomorphism $h$. Hence $\bar{\pi} h$ and $\bar{\pi}$ are isomorphic on $\boldsymbol{P}$.

Ad (c): Let $\bar{f} \in \overline{\boldsymbol{K}}\left(S^{n}, X\right)$ be given then 3.6 ensures the existence of an $r \in \boldsymbol{K}\left(S^{n},|\bar{S}(X)|\right)$ with $[\bar{\omega}][r]^{*}[f]$, hence $\bar{\pi}_{n}(\bar{\omega})$ is an epimorphism. For the proof that $\bar{\pi}_{n}(\bar{\omega})$ is a monomorphism we need a simple lemma: 
Lemma 5.2. (a) A mapping $\bar{f} \in \overline{\boldsymbol{K}}\left(S^{n}, X\right)$ is homotopic to the constant map $0=h(0): S^{n} \rightarrow X$ if and only if $\bar{f}$ has an extension over the $n+1$-cell $E^{n+1} \supset S^{n}$.

(b) For $r \in \boldsymbol{K}\left(S^{n},|\bar{S}(X)|\right)$ we have $\bar{\omega} r \simeq 0$ if and only if $r \simeq 0$.

Proof. Ad (a): Let $g \in \boldsymbol{K}(X, P), P \in \boldsymbol{P}$, then $\bar{f}(g)$ has an extension $F_{g}: E^{n+1} \rightarrow P$. However the definition of a homotopy in $\boldsymbol{K}$ (see 2.3) amounts to the fact that these $F_{g}$ fit together, giving a morphism $\bar{F} \in \overline{\boldsymbol{K}}\left(E^{n+1}, X\right)$ which extends $\bar{f}$.

Ad (b): Due to (a) there exists an extension $\bar{F} \in \overline{\boldsymbol{K}}\left(E^{n+1}, X\right)$ of $\bar{\omega} r$. We consider $\bar{F}$ as a singular $n+1$ simplex and obtain a map $R: E^{n+1} \rightarrow|\bar{S}(X)|$ (the attaching map of $\bar{F}$ as a cell in, $\left.|\bar{S}(X)|\right)$. By assumption one has $\bar{\omega} R=\bar{F}$; and since $r \simeq R \mid S^{n}$ (because $|\bar{S}(X)|$ is the realization of a Kan-set and consequently any $\alpha \in \bar{\pi}_{n}(|\bar{S}(X)|)$ can be represented by a simplicial sphere in dimension $n$ (i.e. a $n$-simplex with completely degenerate boundary) in $\bar{S}(X)$ ) part (a) applies to show $r \simeq 0$. From 5.2 we deduce immediately that $\bar{\pi}_{n}(\bar{\omega})$ is a monomorphism, so 5.1 is thereby proved.

Let $\boldsymbol{E}=\left\{E_{n}, \nu_{n}: \Sigma E \rightarrow E_{n+1}, n \in \mathbf{Z}\right\}$ be a spectrum; we assume henceforth that all $E_{n} \in \boldsymbol{P}$. In [11] G. W. Whitehead defined for any $X \in \boldsymbol{K}$

$$
\begin{aligned}
& X \wedge \boldsymbol{E}=\left\{X \wedge E_{n}\right\} \\
& \bar{\pi}_{n}(\boldsymbol{E})=\lim _{k} \bar{\pi}_{n+k}\left(E_{k}\right)
\end{aligned}
$$

and finally ${ }^{1}$

$$
\bar{H}_{n}(X ; \boldsymbol{E})=\bar{\pi}_{n}(X \wedge \boldsymbol{E}) .
$$

All the ingredients which are necessary for these definitions are available in $\boldsymbol{K}$ as well as in $\overline{\boldsymbol{K}}$, so that we obtain two homology theories $H_{*}$ on $\boldsymbol{K}$ and $\bar{H}_{*}$ on $\overline{\boldsymbol{K}}$. From Theorem 5.1 follows immediately that both these functors are mappings into the category of graded abelian groups. Although $\bar{H}_{*}$ is defined on $\overline{\boldsymbol{K}}$ we can compose it with $h$ and consider it as a homology theory on $\boldsymbol{K}$.

It now makes sense to compare $H_{*}$ with $\bar{H}_{*}$ : They are not isomorphic on $\boldsymbol{K}$; however

1 Actually homology is introduced in [11] by $H_{n}(X ; \boldsymbol{E})=\pi_{n}(\boldsymbol{E} \wedge X)$ which gives of course a functor isomorphic to that defined in (3). 
Proposition 5.3. The functors $H_{*}$ and $\bar{H}_{*}$ are isomorphic on P. Moreover, for $\boldsymbol{E}=\{K(G, n)\}=$ Eilenberg-MacLane spectrum and $G=$ abelian group, $H_{*}(X ; E)=H_{*}(|S(X)| ; G)$ is singular homology, $X \in \boldsymbol{P}$.

Proof. From 4.3 we know that $h$ is compatible with suspension. Therefore (3) determines in both cases isomorphic homology theories. The last statement of 5.3 is implicitly contained in [11]: $H_{*}$ fulfills all the Eilenberg-Steenrod axioms (including the dimensionaxiom for this particular coefficient spectrum) on the category of finite $C W$-complexes. Thus $H_{*}$ is isomorphic to ordinary simplicial homology on this category; and since $H_{*}$ is easily seen to have compact carriers it follows that $H_{*}$ is simplicial homology on $\boldsymbol{P}$ (which on $\boldsymbol{P}$ amounts to the same as singular).

THEOREM 5.4. The transformation $\bar{\omega}:|\bar{S}(X)| \rightarrow X$ induces an isomorphism for all $X \in \boldsymbol{K}$ and all $n$

$$
\bar{H}_{n}(\bar{\omega}): \bar{H}_{n}(|\bar{S}(X)| ; \boldsymbol{E}) \approx \bar{H}_{n}(X ; \boldsymbol{E}) .
$$

Proof. We have

$$
\begin{gathered}
H_{n}(X ; \boldsymbol{E})=\lim _{k} \pi_{n+k}\left(X \wedge E_{k}\right) \\
\bar{H}_{n}(|\bar{S}(X)| ; \boldsymbol{E})=\lim _{k} \bar{\pi}_{n+k}\left(|\bar{S}(X)| \wedge E_{k}\right) .
\end{gathered}
$$

However 4.7 and 3.6 imply

$$
|S(X)| \wedge E_{k} \simeq\left|S\left(X \wedge E_{k}\right)\right|
$$

According to 4.8 this homotopy equivalence is compatible with $\bar{\omega}$. Hence 5.1 (c) gives an isomorphism

$$
\bar{H}_{n}(X ; \boldsymbol{E}) \approx \bar{H}_{n}(|\bar{S}(X)| ; \boldsymbol{E}),
$$

which is easily seen to be that induced by $\bar{\omega}$.

THEOREM 5.5. The transformation $\omega:|S(\cdot)| \mapsto 1_{K}$ induces an isomorphism

$$
H_{n}(\omega): H_{n}(|S(X)| ; \boldsymbol{E}) \approx H_{n}(X ; \boldsymbol{E}) .
$$


Thus (3) is for arbitrary $X \in \boldsymbol{K}$ (not only for $X \in \boldsymbol{P}$ ) isomorphic to singular homology whenever $\boldsymbol{E}$ is an Eilenberg-MacLane spectrum.

The proof is identical with that of 5.4.

THEOREM 5.6. Let $\boldsymbol{E}=\{K(Z, n)\}$ be the Eilenberg-MacLane spectrum for the integers, then there exists a natural transformation

$$
\bar{\eta}_{n}: \bar{\pi}_{n}(X) \longrightarrow \bar{H}_{n}(X ; Z)=\bar{H}_{n}(X)
$$

(the Hurewicz-homomorphism) such that a Hurewicz-theorem holds:

If $\tilde{\pi}_{q}(X)=0$, for $q \leqslant n$, then $\bar{\eta}_{n}: \bar{\pi}_{n}(X) \approx \bar{H}_{n}(X)$ is an isomorphism.

Proof. Let $\epsilon: S^{q} \rightarrow K(Z, q)$ be a fixed map which determines a generator of $Z=\bar{\pi}_{q}(K(Z, q))$ and let $\bar{f} \in \overline{\boldsymbol{K}}\left(S^{n}, X\right)$. Define

$$
\bar{\eta}([\bar{f}])=\left[(\epsilon \wedge 1) \Sigma^{a} \bar{f}\right]
$$

where $(\epsilon \wedge 1) \circ \Sigma^{q} \cdot \bar{f} \in \bar{K}\left(S^{n+q}, X \wedge K(Z, q)\right)$. This yields a natural transformation which behaves in much the same way as the ordinary Hurewicz-homomorphism.

Now for $X=\left(X, x_{0}\right) \in \boldsymbol{K}$, we have the commutative diagram

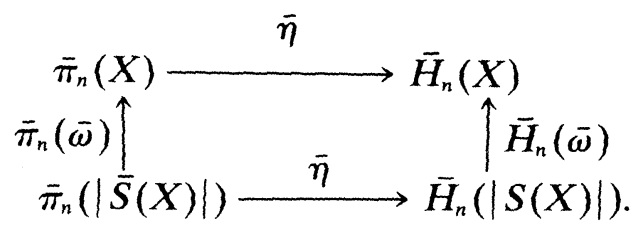

The vertical maps are isomorphisms in view of 5.1 (c). Furthermore one notes that the Hurewicz-homomorphisms $\eta_{n}, \tilde{\eta}_{n}$ in $\boldsymbol{K}$ resp. $\overline{\boldsymbol{K}}$ are compatible with the isomorphisms

$$
\begin{aligned}
& \bar{\pi}_{n}(|\bar{S}(X)|) \approx \bar{\pi}_{n}(|\bar{S}(X)|) \\
& \bar{H}_{n}(|\bar{S}(X)|) \approx H_{n}(|\bar{S}(X)|),
\end{aligned}
$$

since in both categories they are constructed in a completely analogous way. Moreover for $|\bar{S}(X)|$ the assumptions of the classical Hurewicztheorem hold. Hence

$$
\bar{\eta}: \bar{\pi}_{n}(|\bar{S}(X)|) \approx H_{n}(|\bar{S}(X)|)
$$

and all maps in (4) except the upper $\bar{\eta}$ being isomorphisms, this implies 
that $\bar{\eta}$ is also an isomorphism and therefore the Hurewicz-theorem for $\overline{\boldsymbol{K}}$ follows.

Remark. Theorems 5.4, 5.5 confirm that as long as both definitions are feasible, singular homology $H_{n}^{\text {sin }}\left(\right.$ resp. $\left.\bar{H}_{n}^{\text {sin }}\right)$ is in the categories $\boldsymbol{K}$ (resp. $\overline{\boldsymbol{K}}$ ) isomorphic to the Whitehead homology $H_{n}$, (resp. $\left.\bar{H}_{n}\right)$ which has been defined in (3). This fact is not so surprising for $\boldsymbol{K}$ but an interesting phenomenon for $\overline{\boldsymbol{K}}$.

6. A modified singular complex. In this section we introduce a second form of a singular complex $\overline{S^{\prime}}: \overline{\text { Top }} \rightarrow \mathscr{S}_{E}$ which turns out to be more convenient for our purposes.

A singular simplex $\bar{\sigma}^{n} \in \bar{S}^{\prime}(X)$ consists of:

(1) An ordinary singular simplex $\bar{\sigma}^{n} \in \overline{\boldsymbol{K}}\left(\Delta^{n}, X\right)$ in $\bar{S}(X)$.

(2) A rule which assigns a simplicial linear subdivision $\tau_{g}=\tau_{g}\left(\bar{\sigma}^{n}\right)$ of $\Delta^{n}$ to each $g \in \boldsymbol{K}(X, P), P \in \boldsymbol{P}$.

Let $\Delta^{n}$ be the standard $n$-simplex and $\Delta_{i}^{n-1} \subset \Delta^{n}$ a face $i=$ $0, \cdots, n$. A subdivision $\tau_{\imath}$ of $\Delta_{l}^{n-1}$ induces a subdivision of $\Delta^{n}$ in the following way: For each $j$ the order of the vertices of $\Delta_{t}^{n-1}$ and $\Delta_{l}^{n-1}$ determines a linear isomorphism $\varphi_{j}: \Delta_{1}^{n-1} \rightarrow \Delta_{l}^{n-1}$, which transforms to a subdivision $\tau_{j}$ of $\Delta_{\jmath}^{n-1}$; then the cones of the simplexes of all $\Delta_{j}^{n-1}$, $j=0, \cdots, n$ over the barycenter $*$ of $\Delta^{n}$ give a subdivision $\tau$ of $\Delta^{n}$. Note that $\tau$ induces the subdivision $\tau_{1}$ on the given face $\Delta_{l}^{n-1}$, so that this construction gives a degeneracy operator $\tau=s_{t}\left(\tau_{t}\right)$

$$
s_{1}: \bar{S}^{\prime}(X)_{n-1} \longrightarrow \bar{S}^{\prime}(X)_{n}, \quad i=0, \cdots, n .
$$

On the other hand any subdivision $\tau_{g}\left(\bar{\sigma}^{n}\right)$ induces a subdivision of $\Delta_{l}^{n-1}$ for any subsimplex of $\Delta^{n}$. So we also have a face operator $\partial_{\imath}: \bar{S}^{\prime}(X)_{n} \rightarrow \bar{S}^{\prime}(X)_{n-1}$.

Lemma 6.1. The assignment $\bar{S}^{\prime}: \overline{\text { Top }} \rightarrow \mathscr{S}_{E}$ is a 2-functor. The construction of the induced maps and the verification of the 2-functoriality is immediate.

Every $\bar{\sigma}^{n} \in \bar{S}^{\prime}(X)$ is converted into an ordinary singular simplex $\bar{\sigma}^{n} \in \bar{S}(X)$ simply by forgetting the subdivisions of $\Delta^{n}$. This gives a natural functor transformation $p: \bar{S}^{\prime}(X) \rightarrow \bar{S}(X)$. On the other hand one has an inclusion $i: \bar{S}(X) \subset \bar{S}^{\prime}(X)$, by sending each $\sigma^{n} \in \bar{S}(X)$ to the pair $\left(\sigma^{n}, \tau_{g}\right)$, where $\sigma^{n}$ is taken with the identity subdivision. This is also a functor transformation and we have

LEMMA 6.2. There exist natural functor transformations $p: \bar{S}^{\prime} \rightarrow \bar{S}$, $i: \bar{S} \rightarrow \bar{S}^{\prime}$ such that 


$$
p \cdot i=1: \bar{S} \rightarrow \bar{S}
$$

holds.

The main objective of this section is to establish the following assertion:

Proposition 6.3. There exists a natural homotopy

$$
i p \simeq 1: \bar{S}^{\prime} \longrightarrow \bar{S}^{\prime} .
$$

Proof. This homotopy is constructed inductively. For $n=0$ we have $\bar{S}^{\prime}(X)_{0}=\bar{S}(X)_{0}$; let us assume that a homotopy $\lambda_{k}$ : ip $\simeq 1$ has been already established on the $k$-skeleton for $k \leqq n-1$.

Let $\bar{\sigma}^{n} \in \bar{S}^{\prime}(X)_{n}, g \in \boldsymbol{K}(X, P), P \in \boldsymbol{P}$ and * the "barycenter" of $\Delta^{n} \times I$, be given. The boundary of $\Delta^{n} \times I$ has received a triangulation, consisting of $\tau_{g}\left(\bar{\sigma}^{n}\right)$ on $\Delta^{n} \times 0$, the identical subdivision on the top $\Delta^{n} \times 1$ (i.e. the subdivision of $i p \bar{\sigma}^{n}$ ) and by the inductive hypothesis some subdivision on $\left(b d \Delta^{n} \times I\right)$. We now obtain a triangulation $\tau_{g}$ of $\Delta^{n} \times I$ by forming the cones over all simplexes of $b d\left(\Delta^{n} \times I\right)$ with top vertex *. There is a mapping $\rho^{n}: \Delta^{n} \times I \rightarrow P$ which is induced by $p \bar{\sigma}^{n}$ and $\lambda_{n-1}$. Ordinary semi-simplicial techniques and the definition of semisimplicial homotopy can be used to establish a homotopy $\lambda_{n}: i p \simeq 1$ on the $n$-skeleton of $\bar{S}^{\prime}(X)$.

Proposition 6.3 is thereby proved.

By combining 6.2, 6.3 we have established

THEOREM 6.4. The functors $\overline{\boldsymbol{S}}, \overline{\boldsymbol{S}}^{\prime}: \overline{\boldsymbol{K}} \rightarrow \mathscr{S}_{E}$ are homotopy equivalent. Hence $\bar{S}(X)$ and $\bar{S}^{\prime}(X)$ as well as $\left\{\bar{S}(X) \mid\right.$ and $\left|\bar{S}^{\prime}(X)\right|(X \in K)$ are homotopy equivalent.

7. Comparison of $\overline{\boldsymbol{H}}_{*}$ and the SteenrodSitnikov-homology. In 1940, N. Steenrod introduced [10] a homology theory which has become of increasing importance in topology. In 1960 J. Milnor gave an axiomatic description of Steenrod's homology functor $H_{*}^{S}$ on the category of compact metric spaces [8]. Many important results on $H_{*}^{s}$ have been established in the last years (e.g. by K. Sitnikov [1], Sklarjenko and others). In this paper we do not intend to give an explicit construction of $H_{*}^{s}$ but we will use the following axiomatic description which stems from [8]:

THEOREM 7.1. The homology functor on the category of pairs of compact metric spaces $\mathbf{C o m}^{2}$ is up to an isomorphism characterized by the Eilenberg-Steenrod axioms and the two additional axioms 
(A) (Strong excision). If $f:(X, A) \rightarrow(Y, B)$ is a relative homeomorphism (i.e. $f \mid(X-A): X-A \rightarrow Y-B$ is a homeomorphism) then $H_{*}^{s}(f): H_{*}^{s}(X, A) \rightarrow H_{*}^{s}(Y, B)$ is an isomorphism.

(B) (Cluster axiom). Let $X, X_{\imath}, i=1,2, \cdots$ be compact metric spaces with $X=\bigcup_{1}^{\infty} X_{i}, \quad X_{1} \cap X_{1}=*$ (=basepoint) $i \neq j$ and with $\lim d\left(X_{i}\right)=0(d(A)=$ diameter of $A)$. Then the projections $p_{i}: X \rightarrow X_{t}$ induce an isomorphism $H_{*}^{S}(X) \rightarrow \prod_{i=1}^{\infty} H_{*}^{S}\left(X_{i}\right)$.

In the following we take integer coefficients.

To turn $\bar{H}_{*}$ (from $\$ 5(3)$ ) into a homology theory on $\mathbf{C o m}^{2}$, we will use the Eilenberg-MacLane spectrum $\boldsymbol{E}$. In order to get a functor $\bar{H}_{*}: \mathbf{C o m}^{2} \rightarrow$ graded groups we follow the classical line and define for the pair $(X, A)$ where $A \subset X$

$$
\bar{H}_{n}(X, A)=\bar{H}_{n}(X / A, E) .
$$

For this we assume that the subcategory $\boldsymbol{P} \subset \boldsymbol{K}=\mathbf{C o m}$ is the full subcategory of all connected (consequently also pathwise connected) $C W$-spaces.

Since $X$ and $A$ are compact metric, we observe that

(1): $(X / A *)$, where $*$ is the basepoint stemming from $A$, is simply the Alexandroff compactification of $X-A$ and is metrizable, and (2): each relative homeomorphism $f:(X, A) \rightarrow(Y, B)$ therefore induces a homeomorphism $f^{*}:(X / A, *) \rightarrow(Y / B, *)$. This implies holds.

Proposition 7.2. For $\bar{H}_{*}$ on $\mathbf{C o m}^{2}$ the strong excision axiom (A)

The verification of the Eilenberg-Steenrod axioms for $\bar{H}_{*}$ depends upon the following

Lemma 7.3. For any pair $(X, A) \in \mathbf{C o m}^{2}$ there exists a natural chain homotopy equivalence

$$
C_{*}(X, A) \simeq C_{*}(X) / C_{*}(A)
$$

where $C_{*}(Y)=C_{*}\left(\bar{S}^{\prime}(Y)\right), Y \in \boldsymbol{K}$.

The proof will be given in the next section.

It follows from 7.3 that 
Proposition 7.4. The functor $\bar{H}_{*}$ fulfills the Eilenberg-Steenrod axioms.

The proof follows the classical model closely: The homotopy axiom is true because we repeat the usual argument that a homology theory on a category of based spaces leads to a homology theory (which fulfills the homotopy axiom) on a category of pairs. The excision axiom is contained in 7.2. Since a point is an object of $\boldsymbol{P}$, the dimension axiom follows from 5.3. What remains is the exactness. But Lemma 7.3 has the following interpretation: One can exhibit $\bar{H}_{*}(X / A)$ as the homology of the chain complex $C_{*}(X) / C_{*}(A)$. Since we have an exact sequence of chain complexes

$$
0 \rightarrow C_{*}(A) \stackrel{i}{\rightarrow} C_{*}(X) \rightarrow C_{*}(X / A) \rightarrow 0
$$

( $i: A \subset X$ the inclusion, $p$ the projection), the exactness of $\bar{H}_{*}$ then follows from homological algebra.

Proposition 7.4 is thereby proved.

We now turn to the cluster axiom (B) in 7.1. To this end we need some preliminary constructions:

Let $a_{i}, i=0, \cdots, n$ be the unit point on the axes in $R^{n+1}$, and let $\Delta^{n}=\left(a_{0}, \cdots, a_{n}\right)$ be the standard simplex spanned by these points. For each $k$ belonging to $\mathbf{Z}^{+}$, let $\Delta_{k}^{n}$ be the simplex $1 / k \Delta^{n}=\left(1 / k a_{0}, \cdots, 1 / k a_{n}\right)$ and let $\Sigma^{n}=([0,1], 0, \cdots, 0) \cup \bigcup_{k \geqq 1} \Delta_{k}^{n}$. With the subspace topology, $\Sigma^{n}$ is compact metric. We denote by

$$
\gamma_{k}=\left(\left[\frac{1}{k+1}, \frac{1}{k}\right], 0,-, 0\right)
$$

the segment joining the first vertex of $\Delta_{k}^{n}$ with that of $\Delta_{k+1}^{n}$, and let

$$
\Sigma_{k}^{n}=\Sigma^{n} \cap\left\{x \in \mathbf{R}^{n+1} \mid x_{i} \geqq \frac{1}{k}, i=0, \cdots, n\right\} .
$$

Let $\Delta^{n}$ be the standard simplex in another $\mathbf{R}^{n+1}$. We shall construct surjections $r_{k}: \Delta^{n} \rightarrow \Sigma_{k}^{n}$ and $r: \Delta^{n} \rightarrow \Sigma^{n}$. For each $i \in \mathbf{Z}^{+}$let $T_{i}$ be the zones

$$
\left\{x \in \Delta^{n} \mid 1-\frac{1}{i} \leqq x_{0} \leqq 1-\frac{1}{i+1}\right\}
$$


and map $T_{2{ }_{\imath-1}}$ onto $\Delta_{i}^{n}$ by a homeomorphism relative to the boundary and each $T_{2 i}$ onto $\gamma_{\iota}$.

Defining $r\left(a_{0}\right)=(0, \cdots, 0)$, the origin, gives us the desired $r: \Delta^{n} \rightarrow \Sigma^{n}$. Stopping the above construction with $T_{2_{t-1}}$ and mapping everything remaining onto $\Delta_{k}^{n}$ by a $P L$-homeomorphism gives us the required $r_{k}: \Delta^{n} \rightarrow \sum_{k}^{n}$.

We now apply this construction to singular homology: Let $X$ be any path connected space and $c=\Sigma r_{i} \sigma_{i}^{n}, r_{i} \in \mathbf{Z}$ be any singular $n$-chain ${ }^{2}$ in $X$. Letting $|c|=\Sigma\left|r_{t}\right|$ we define a map $\beta_{|c|}: \Sigma_{|c|}^{n} \rightarrow X$ as follows: We map at first the $\left|r_{1}\right|$ copies of $\Delta^{n}$ in $\Sigma_{|c|}^{n}$ into $X$ by

$$
\beta_{|c|} \mid \Delta_{\imath}^{n}=\left\{\begin{array}{rcc}
\sigma_{1}^{n} & \text { if } & r_{1}>0 \\
-\sigma_{1}^{n} & \text { if } & r_{1}<0
\end{array} \quad i=1, \cdots, r_{1}\right.
$$

where $-\sigma_{1}^{n}$ means $\sigma_{1}^{n}$ after a change of orientation (permute the first two vertices of $\Delta^{n}$ ) and proceed analogously for the next $\left|r_{2}\right|,\left|r_{3}\right| \cdots$ etc. copies of $\Delta^{n}$. Since $X$ is path-connected we can extend $\beta_{|c|}$ over whole $\Sigma_{|c|}^{n}$. These constructions allow us to replace each singular chain $\bar{c} \in$ $C_{*}\left(\bar{S}^{\prime}(X)\right), X \in \mathbf{C o m}$, by a single singular simplex $\phi(c)$ : In fact let $\bar{c}=\Sigma \alpha_{\imath} \bar{\sigma}_{\imath}^{n}, \alpha_{\imath} \in \mathbf{Z}$ and $g \in \boldsymbol{K}(X, P), P \in \boldsymbol{P}$, then each simplex $\Delta_{k}^{n}$ of $\Sigma_{|c|}^{n}$ inherits a subdivision from $\bar{\sigma}_{i}^{n}(g)$ and this determines a triangulation $\tau_{g}$ of $\Sigma_{|c|}^{n}$ (which depends on $g$ ). Denote by $\rho_{g}$ a subdivision of $\Delta^{n}$ which makes $r_{|c|}: \Delta^{n} \rightarrow \Sigma_{|c|}^{n}$ (the latter with the triangulation $\tau_{g}$ ) simplicial. Then the mapping

$$
\langle\bar{c}\rangle(g): \Sigma_{|c|}^{n} \rightarrow P
$$

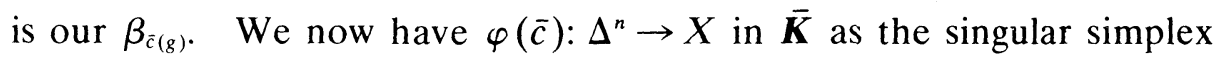
$\varphi(\bar{c})(g)=\langle\bar{c}\rangle(g) r_{|c|}$ with subdivision $\rho_{g}$ for any given $g \in \boldsymbol{K}(X, P) P \in$ $\boldsymbol{P}$. This determines a singular simplex in $\bar{S}^{\prime}(X)$. Furthermore the mapping cylinder of $r_{|c|}$ can be mapped into $P$ so that there exists a chain họmotopy $s$ between $\varphi(\bar{c})$ and $\bar{c}$.

Summarizing, we have established

LEMMA 7.5. There exists a chain homotopy

$$
s: \varphi \simeq 1: C_{*}\left(\bar{S}^{\prime}(X)\right) \longrightarrow C_{*}\left(\bar{S}^{\prime}(X)\right) .
$$

Now let $X$ be a cluster consisting of spaces $X_{t}$ as in $7.1(\mathrm{~B})$ and let $\left.\pi=\Pi_{1}^{\infty} p_{\imath}: \quad C_{*}\left(\bar{S}^{\prime}(X)\right) \rightarrow \Pi=\Pi_{1}^{\infty} C_{*}\left(\bar{S}^{\prime} X_{\imath}\right)\right)$ where $p_{\imath}: X \rightarrow X_{\iota}$ is the

\footnotetext{
${ }^{2}$ We use ordinary singular homology for this construction.
} 
projection. An element $f=\left\{\bar{c}_{i}\right\} \in \Pi$ is a sequence, $\bar{c}_{i} \in C\left(\bar{S}^{\prime}\left(X_{i}\right)\right)$. We set $z_{j}=\sum_{i=1}^{i} c_{l}$; because $\Sigma^{n}=\bigcup_{i=1} \sum_{\left|z_{i}\right|}^{n}, \sum_{\left|z_{i}\right|}^{n} \subset \Sigma_{\left|z_{i+1}\right|}^{n}$, by defining for each $g \in \boldsymbol{K}(X, P), P \in \boldsymbol{P}$ a map $f_{g}: \Sigma^{n} \rightarrow P, f_{g} \mid \Sigma_{\left|z_{i}\right|}^{n}=\left\langle z_{i}\right\rangle(g)$, we obtain a consistently defined map $\Sigma^{n}-X$.

Because $P$ is locally contractible, there is a neighborhood $U$ of the basepoint $* \in P$ that is contractible and there exists an index $N$ such that

$$
\bigcup_{1 \geqq N} \sum_{\left|z_{1}\right|}^{n}
$$

is mapped into $U$.

Thus the mapping $f_{g} r: \Delta^{n} \rightarrow P, r: \Delta^{n} \rightarrow \Sigma^{n}$ is homotopic to a map $f_{g} i_{k} r_{k}=\varphi\left(z_{k}\right)(g)$, where $r_{k}: \Delta^{n} \rightarrow \sum_{k}^{n}$ is as above and

$$
i_{k}: \sum_{k}^{n} \subset \Sigma^{n}
$$

is the inclusion. We have a triangulation $\rho_{g}$ of $\Delta^{n}$ which is obtained in the same way as before. The number $k=\left|z_{N}\right|$ depends on $g$ and in particular on the choice of $U$.

We intend to define a singular simplex $\varphi(f) \in \bar{S}^{\prime}(X)$ by $\varphi(f)(g)=$ $\varphi\left(z_{k}\right)(g)$ and the triangulation $\rho_{g}$.

For $t \in \boldsymbol{P}\left(P_{1}, P_{2}\right),(t, \omega): g_{1} \rightarrow g_{2}, g_{\imath} \in \boldsymbol{K}\left(X, P_{t}\right)$ we have a homotopy $\omega^{\prime}: t\left\langle z_{k_{1}}\right\rangle\left(g_{1}\right) \simeq\left\langle z_{k_{2}}\right\rangle\left(g_{2}\right)$ (where of course $k_{i}$ belongs to $g_{i}$ resp. to the neighborhoods $U_{1}$ of the basepoints in $P_{i}$ ) and the corresponding deformations of $f_{g} \mid \bigcup_{i \geqq N} \sum_{\left|z_{i}\right|}^{n}$ for sufficiently large $N$ (e.g. $N=$ $\left.\max \left(k_{1}, k_{2}\right)\right)$ to the constant map. We can now repeat the arguments in $\S 3$ which lead to a proof of Theorem 3.8 resp. 3.10 and apply Lemmas 3.9, 3.11 in the present situation to show that $\varphi(f)$ can be made functorial (the necessary tree category is in all cases the same). Thus $\varphi(f)$ becomes a map in $\overline{\boldsymbol{K}}\left(\Delta^{n}, X\right)$ and we obtain a singular simplex $\varphi(f) \in \bar{S}^{\prime}(X)$.

We now prove the existence of a chain homotopy

$$
p_{j} \cdot \phi \simeq q_{j}
$$

where $q_{j}(f)=q_{j}\left(\left\{c_{l}\right\}\right)=c_{j}$. To this end let $g \in \boldsymbol{K}(X, P), P \in \boldsymbol{P}$ be an arbitrary map. If $k(g) \geqq j$, then $p_{j} \cdot \phi=q_{j}$; if $k(g)<j$, the contraction homotopy of $U$ into the basepoint yields a chain homotopy $s: p_{j^{*}} \phi \simeq q_{j}$. This can again be made functorial ${ }^{3}$ by using 3.9, 3.11. Now (4) is easily seen to give a chain homotopy

${ }^{3}$ In this particular case we can take into account that $\varphi$ has been given originally also on the iubcategory $\boldsymbol{P}_{X}^{\prime}$. The extensions of $s$ and $\varphi$ from $\boldsymbol{P}_{X}^{\prime}$ to $\boldsymbol{P}_{X}$ using the techniques of Lemma 3.9 and .11 are therefore compatible with each other (i.e. the corresponding functors fit gether). However the reader will easily realize that the proof in this case (as well as in all similar ses which follow) can be established without using this fact. 
In order to prove that $\varphi \pi$ is also homotopic to 1 , we make the following observations:

Let $\bar{c} \in C_{*}\left(\bar{S}^{\prime}(X)\right), \quad \pi(\bar{c})=f=\left\{p_{i} \cdot \bar{c}\right\}=\left\{\bar{c}_{i}\right\}, \quad g \in \boldsymbol{K}(X, P), \quad P \in \boldsymbol{P}$ then we have chain homotopies (for different reasons, partly because of 7.5 , partly because of the contraction of the neighbourhood $U$ in $P$ )

$$
\varphi(f)(g)=\varphi\left(z_{k}\right)(g) \simeq z_{k}(g) \simeq \bar{c}(g)
$$

where $k=k(g)$ and $z_{k}=\sum_{k=1}^{l} \bar{c}_{i}$ are as before. This leads immediately (using Lemma 3.9, Lemma 3.11 and the related arguments) to a chain homotopy

$$
s: \varphi \pi \simeq 1
$$

Now we can establish

Proposition 7.6. For the homology functor $\bar{H}_{*}$ the cluster axiom 7.1 (B) holds.

Proof. The homology of the chain complex $\Pi=\Pi_{1}^{\infty} C_{*}\left(\bar{S}^{\prime}(X)\right)$ is, due to Theorems $7.5,6.4$

$$
H_{*}(\Pi)=\prod_{1}^{\infty} \bar{H}_{*}\left(X_{\imath}\right)
$$

However (5), (6) imply that $\pi$ induces an isomorphism in homology.

This leads to

THEOREM 7.7. On the category $\boldsymbol{K}=\mathbf{C o m}, P=$ subcategory of connected $C W$-spaces in $\boldsymbol{K}$, there exists a natural isomorphism

$$
\bar{H}_{*} \approx H_{*}^{s} .
$$

Since the homology functors $\bar{H}_{*}, H_{*}^{S}$ can be considered as functors on $\mathbf{C o m}^{2}$ (we have used this fact in the proof of Theorem 7.7), the following corollary (which refers to the category of pairs) holds:

Corollary 7.8. Let $\bar{H}_{*}$ on $\mathbf{C o m}^{2}$ be singular homology (in the sense of (1)) with integer coefficients (i.e. $\left.\bar{H}_{*}(X, Y)=\bar{H}_{*}(X / Y)\right)$ and $H_{*}^{s}$ Steenrod-Sitnikov homology (for example in the form of [8]), then both functors are isomorphic. 
REMARK. K. Sitnikov [1] extended $H_{*}^{S}$ to arbitrary metric spaces simply by taking compact carriers. Our $\bar{H}_{*}$ does not necessarily have compact carriers if $\boldsymbol{K}=$ Top and $\boldsymbol{P}=$ category of connected $C W$-spaces.

8. Proof of Lemma 7.3. The idea of the proof of Lemma 7.3 is this: At first apply Lemma 7.5 to the effect that a given chain $\bar{c} \in C_{*}(X / A)$ is converted into a singular simplex $\bar{\sigma}=\varphi(\bar{c})$. Now we modify $\bar{\sigma}$ to a singular chain $\bar{c}_{1}$ in $C_{*}\left(\bar{S}^{\prime}(X)\right)$ (simply by cutting out the counterimage of the basepoint $* \in P / Q$ in (1) for any $g$ ) and apply again Lemma 7.5. This gives a singular simplex $\psi(\bar{\sigma})=\psi \varphi(\bar{c})=\beta(\bar{c}) \in$ $\bar{S}^{\prime}(X) / \bar{S}^{\prime}(A)$ and finally chain homotopies

$$
\alpha \beta \simeq 1, \quad \beta \alpha \simeq 1
$$

We have the diagram

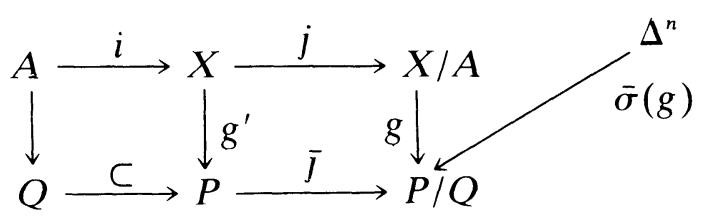

with $\bar{\sigma} \in \bar{S}^{\prime}(X / A), P, Q \in \boldsymbol{P}$ and denote the counterimage of the basepoint $* \in P / Q$ in $\Delta^{n}$ (under the mapping $\bar{\sigma}(g)$ ) by $T$. Certainly one has $\bar{j}^{-1}(*)=Q$.

We distinguish two different cases: For $\bar{\sigma}(g) \equiv *(=$ constant map onto the basepoint) we define

$$
\psi(\bar{\sigma})\left(g^{\prime}\right) \equiv *
$$

where now $*$ denotes a fixed base point in $Q \subset P$.

In the remaining case we have $\bar{\sigma}(g) \supsetneqq *$. Let $W^{\prime} \supset Q$ be a neighborhood which can be deformed onto $Q, W$ its image under $\bar{J}$ and $T=\bar{\sigma}(g)^{-1}(*) \subset \Delta^{n}$. We can find a neighborhood $U$ of $T$ such that $\bar{\sigma}(g)(U) \subset W$ and a subdivision $\tau_{g}^{\prime}$ of the given triangulation $\tau_{g}$ of $\Delta^{n}$ such that every closed simplex which intersects $T$ is contained in $U$. The subchain of $\Delta^{n}$ in this triangulation, which consists of all simplexes which are contained in $U$ (with orientation inherited from $\Delta^{n}$ ) is denoted by $t^{n}$, the chain consisting of all remaining simplexes of $\tau_{g}^{\prime}$ by $u^{n}$. These chains induce under $g$ a singular chain $u^{n}(g)$ resp. $t^{n}(g) \in$ $C_{*}(S(P / Q))$.

Moreover there exists by construction a counterimage $v^{n}\left(g^{\prime}\right)$ of $u^{n}(g)$ under $\bar{j}_{*}: C_{*}(S(P)) \rightarrow C_{*}(S(P / Q))$ which after the application of 
the deformation $d^{\prime}: W^{\prime} \rightarrow Q$ becomes a chain $u^{n}\left(g^{\prime}\right) \in$ $C_{*}(S(P)) / C_{*}(S(Q))$. We can convert $u^{n}\left(g^{\prime}\right)$ into a singular simplex, obtaining the simplex $\psi(\bar{\sigma})\left(g^{\prime}\right)$.

Let $(r, \omega): g_{1} \rightarrow g_{2}$ be a morphism in $\boldsymbol{P}_{X}$ and assume that $\omega: r g_{1} \simeq g_{2}$ is a homotopy (c.f. $\S 10) H: \Delta^{n} \times I \rightarrow P_{2}$, then we can replace $\Delta^{n}$ by $\Delta^{n} \times I$ in the preceding construction and obtain a homotopy $\omega^{\prime}: r \circ \psi(\bar{\sigma})\left(g_{1}\right) \simeq$ $\psi(\bar{\sigma})\left(g_{2}\right)$ in precisely the same way as we got $\psi(\bar{\sigma})$ from $\bar{\sigma}$. In case $H \equiv *$ is the trivial homotopy, we take for $\omega^{\prime}$ the trivial homotopy. This enables us to construct a singular simplex $\psi(\bar{\sigma}) \in \bar{S}^{\prime}(X)$ by recalling the fact that $\boldsymbol{P}_{X}$ is a tree category ( $\$ 1$ (e) and application of Lemma 3.9, 3.11).

In order to complete the proof of Lemma 7.3, we need chain homotopies

$$
\begin{aligned}
& \beta \alpha \simeq 1: C_{*}(X) / C_{*}(A) \longrightarrow C_{*}(X) / C_{*}(A) \\
& \alpha \beta \simeq 1: C_{*}(X / A) \longrightarrow C_{*}(X / A) .
\end{aligned}
$$

For this purpose observe that for any $\bar{\sigma}^{n} \in C_{n}(X), g^{\prime} \in \boldsymbol{K}(X, P)$ we have a decomposition

$$
\bar{\sigma}^{n}\left(g^{\prime}\right)=v^{n}\left(g^{\prime}\right)+t^{n}
$$

where $t^{n}$ is a singular chain in $W^{\prime}$ and $v^{n}\left(g^{\prime}\right)$ is a singular chain in $S(P) / S\left(W^{\prime}\right)$. The deformation $d^{\prime}: W^{\prime} \rightarrow Q$ provides us with a chain homotopy

$$
\psi \alpha\left(\bar{\sigma}^{n}\right)\left(g^{\prime}\right)=u^{n}\left(g^{\prime}\right) \simeq v^{n}\left(g^{\prime}\right)+t^{n}=\bar{\sigma}^{n}\left(g^{\prime}\right)
$$

Using the standard techniques we obtain a chain homotopy (3).

Let $\tau^{n} \in \bar{S}^{\prime}(X / A)$, then we have

$$
\tau^{n}(g)=u^{n}(g)+t^{n}(g)
$$

where $u^{n}(g)$ resp. $t^{n}(g)$ is induced by the preceding construction of $u^{n}$, resp. $t^{n}$ as subchains of $\Delta^{n}$ as above.

From (6) we deduce

$$
\alpha \psi\left(\tau^{n}\right)(g)=u^{n}(g) \simeq u^{n}(g)+t^{n}(g)
$$

where the last homotopy equivalence stems from the deformation $d: W \rightarrow * \in P / Q$.

Again this leads to a chain homotopy between $\alpha \psi\left(\tau^{n}\right)$ and $\tau^{n}$ which finally gives the chain homotopy (4).

This completes the proof of Lemma 7.3. 


\section{REFERENCES}

1. P. S. Alexandrov, Topological duality theorems (II) (non-closed sets) Publ. of the Stekloff Institute, Sowj. Akad. of Sc. Vol. LIV Moscow (1959).

2. F. W. Bauer, Über Fortsetzungen von Homologiestrukturen, Math. Ann. Bd. 135 (1958), 93-114.

3. _- Homotopietheorie B. I. Taschenbücher, 475 Mannheim (1971).

4. Stabile Kategorien, Math. Z. Bd. 123 (1971), 139-167.

5. J. Dugundji, Topology, Allyn \& Bacon, Inc. Boston (1966).

6. P. Gabriel and M. Zisman, Calculus of fractions and homotopy theory, Springer Verlag Berlin (1966).

7. S. Mardéšic, Shapes of topological spaces, Proceedings of the Hungarian topology conference, Keszthely (1972).

8. J. Milnor, On the Steenrod homology theory, (mimeographed notes) Berkeley (1960).

9. L. D. Mzinarishvili, On the equivalence of the Kolomogoroff and the Steenrod homology, Publ. of the Mathm. Institute of the Georgian Acad. of Sc. (Collected papers on homology) (1972), 143-163.

10. N. Steenrod, Regular cycles of compact metric space, Ann. of Math., 41 (1940), 833-851.

11. G. W. Whitehead, Generalized homology theories, Trans. of Amer. Math. Soc., 102 (1962), $227-283$.

Received May 3, 1974.

UNIVERSITÄT FRANKFURT

Math Seminar 




\section{Pacific Journal of Mathematics}

Vol. 64, No. 1

May, 1976

Walter Allegretto, Nonoscillation theory of elliptic equations of order $2 n \ldots \ldots \quad 1$

Bruce Allem Anderson, Sequencings and starters.................. 17

Friedrich-Wilhelm Bauer, A shape theory with singular homology .......... 25

John Kelly Beem, Characterizing Finsler spaces which are

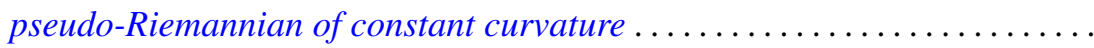

Dennis K. Burke and Ernest A. Michael, On certain point-countable

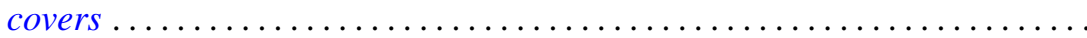

Robert Chen, A generalization of a theorem of Chacon ............... 93

Francis H. Clarke, On the inverse function theorem ................ 97

James Bryan Collier, The dual of a space with the Radon-Nikodým

property ....................................... 103

John E. Cruthirds, Infinite Galois theory for commutative rings ............ 107

Artatrana Dash, Joint essential spectra......................... 119

Robert M. DeVos, Subsequences and rearrangements of sequences in FK

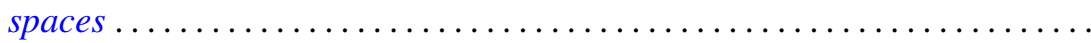

Geoffrey Fox and Pedro Morales, Non-Hausdorff multifunction generalization

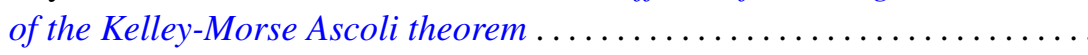

Richard Joseph Fleming, Jerome A. Goldstein and James E. Jamison, One

parameter groups of isometries on certain Banach spaces.............

Robert David Gulliver, II, Finiteness of the ramified set for branched

immersions of surfaces

Kenneth Hardy and István Juhász, Normality and the weak cb property ...... 167

C. A. Hayes, Derivation of the integrals of $L^{(q)}$-functions.

Frederic Timothy Howard, Roots of the Euler polynomials .

Robert Edward Jamison, II, Richard O'Brien and Peter Drummond Taylor, On

embedding a compact convex set into a locally convex topological vector space ....................................

Andrew Lelek, An example of a simple triod with surjective span smaller than span ...

Janet E. Mills, Certain congruences on orthodox semigroups

Donald J. Newman and A. R. Reddy, Rational approximation of $e^{-x}$ on the positive real axis.

John Robert Quine, Jr., Homotopies and intersection sequences ...

Nambury Sitarama Raju, Periodic Jacobi-Perron algorithms and fundamental units ....

Herbert Silverman, Convexity theorems for subclasses of univalent functions. . .

Charles Frederick Wells, Centralizers of transitive semigroup actions and endomorphisms of trees.........................

Volker Wrobel, Spectral approximation theorems in locally convex spaces ..................... 